\title{
Neuroprotective Effects of Curcumin-Loaded Emulsomes in a Laser Axotomy-Induced CNS Injury Model
}

This article was published in the following Dove Press journal: International Journal of Nanomedicine

\author{
Elif Nur Yilmaz $\mathbb{D}^{1,2}$ \\ Sadik Bay iD ' \\ Gurkan Ozturk (iD) 1,3 \\ Mehmet Hikmet Ucisik (iD) 1,4 \\ 'Regenerative and Restorative Medicine \\ Research Center (REMER), Research \\ Institute for Health Sciences and \\ Technologies (SABITA), Istanbul Medipol \\ University, Beykoz, Istanbul, Turkey; \\ ${ }^{2}$ Graduate School of Engineering and \\ Natural Sciences, Istanbul Medipol \\ University, Beykoz, Istanbul, Turkey; \\ ${ }^{3}$ Department of Physiology, International \\ School of Medicine, Istanbul Medipol \\ University, Beykoz, Istanbul, Turkey; \\ ${ }^{4}$ Department of Biomedical Engineering, \\ School of Engineering and Natural \\ Sciences, Istanbul Medipol University, \\ Beykoz, Istanbul, Turkey
}

Correspondence: Mehmet Hikmet Ucisik Department of Genetics and Bioengineering, Faculty of Engineering, Yeditepe University, 34755 Istanbul Turkey

Tel $+902165780000-3259$

Fax +902165780829

Email m.h.ucisik@gmail.com
Purpose: Curcumin, a polyphenol isolated from the rhizomes of turmeric, holds great potential as a neuroprotective agent in addition to its anti-inflammatory and antioxidant characteristics. The poor bioavailability and low stability of curcumin are the greatest barriers to its clinical use. This study aims to investigate the neuroprotective effect of curcumin on axonal injury, by delivering the lipophilic polyphenol to a primary hippocampal neuron culture by means of a lipid-based drug delivery system, named emulsomes.

Methods: To study neuroregeneration ex vivo, an injury model was established through single-cell laser axotomy on hippocampal neurites. Upon treatment with curcumin-loaded emulsomes (CurcuEmulsomes), curcumin and CurcuEmulsome uptake into neurons was verified by three-dimensional Z-stack images acquired with confocal microscopy. Neuron survival after axonal injury was tracked by propidium iodide (PI) and Hoechst staining. Alterations in expression levels of physiological markers, such as anti-apoptotic marker $\mathrm{Bcl} 2$, apoptotic marker cleaved caspase 3, neuroprotective marker Wnt3a and the neuronal survival marker mTOR, were investigated by immunocytochemistry analyses.

Results: The results indicated significant improvement in the survival rate of injured neurons upon CurcuEmulsome treatment. Bcl2 expression was significantly higher for injured neurons treated with curcumin or CurcuEmulsome. Reduction in caspase 3 expression was seen in both curcumin and CurcuEmulsome treatment, whereas there were no significant changes in Wnt3a and mTOR expression.

Conclusion: The established laser-axotomy model was proven as a reliable methodology to study neurodegenerative models ex vivo. CurcuEmulsomes delivered curcumin to primary hippocampal neurons successfully. Treated with CurcuEmulsomes, injured hippocampal neurons benefit from the neuroprotective effects of curcumin, exhibiting a higher survival rate and increased anti-apoptotic marker levels.

Keywords: curcumin, lipid-based nanodrug delivery, neurodegeneration, hippocampus, axon injury

\section{Introduction}

As the demographics in developed countries shift towards an aging population, neurodegenerative diseases (NDs) including Alzheimer's disease (AD), amyotrophic lateral sclerosis (ALS) and Parkinson's disease (PD) are predicted by the World Health Organization (WHO) to be the second most common cause of death following cardiovascular disease by $2040 .{ }^{1}$ NDs are characterized by progressive loss of function and structure of neurons ending with neuronal death. ${ }^{2-4}$ Axon 
degeneration, one of the common hallmarks of both nerve injury and neurodegenerative diseases, causes permanent disability. ${ }^{5,6}$ Axons are the first neuronal components to become damaged, and degeneration of axons may begin many years before the eventual death of the cell. ${ }^{7,8}$ Although axon regeneration is necessary for neuronal survival, there are no effective treatments to inhibit axonal degeneration. ${ }^{6}$

Axotomy is an experimental model of neuronal injury and subsequent cell death. Axotomy is widely applied in research to study the biological response of neurons and axons from an injury established via transection of the neurons' axon in vitro. Activation of neuroprotective pathways and sustainment of endogenous brain neurogenesis are regarded as therapeutic approaches to convert neurodegeneration to neuroregeneration. ${ }^{9}$

Current challenges with neuroprotective therapeutic approaches include their high cost, lack of efficacy, drug resistance and certain side effects. ${ }^{10,11}$ As an alternative, natural biomolecules have stimulated renewed interest in recent years. For instance, curcumin, a polyphenol isolated from the rhizome Curcuma longa, is a biologically active compound shown to play a role as a neuroprotective agent in $\mathrm{AD}, \mathrm{PD}$, ischemic stroke and alcohol-induced neurodegeneration models. ${ }^{12-25}$ Curcumin's diverse biological functions include antioxidation, anti-inflammation, neuroprotection, and protection of mitochondrial and bloodbrain barrier (BBB) integrity. ${ }^{12,26-36}$ Thus, given alone or combined with other neuroprotective agents, curcumin may be beneficial as a therapy for neurodegenerative diseases. ${ }^{37,38}$

However, the medical use of curcumin is limited by its poor bioavailability and low stability. Curcumin exhibits very low water solubility (ie $11 \mathrm{ng} / \mathrm{mL}$ ), ${ }^{39-44}$ poor absorption, ${ }^{44}$ rapid metabolism ${ }^{40,45,46}$ and fast systemic elimination. ${ }^{23}$

Drug delivery systems are considered the prominent solution to enhance the solubility, stability and cellular uptake efficacy of curcumin. ${ }^{47}$ Delivery of curcumin within various nanocarriers has been shown to increase curcumin penetration through the BBB and sustain curcumin release inside the central nervous system (CNS). ${ }^{48-50}$

In this study, a recently developed lipid-based nanoparticular system, curcumin-loaded emulsomes, or so-call ed CurcuEmulsomes, ${ }^{51}$ are used to enable delivery of curcumin to primary hippocampal neuron culture. To study the neuroregenerative effect of the formulation, an axonal injury model was successfully established ex vivo through laser axotomy on the neurites. Curcumin-loaded emulsomes are investigated here for the first time as a neuroprotective nanomedicine on a novel, repetitive and robust neuron injury model.

\section{Methods}

\section{Animals}

Hippocampal neurons for in vitro studies were isolated from newborn BALB/c mice (Regenerative and Restorative Medicine Research Center [REMER], Istanbul, Turkey) on postnatal day zero (P0). Animal experiments were conducted in accordance with the European Community guidelines and approved by Istanbul Medipol University Ethics Committee for Animal Experimentation (date: 07/03/2016; approval number: 38828770-604.01.01-E.3722).

\section{Chemicals and Reagents}

Curcumin (purity $\geq 95 \%$ ), glyceryl tripalmitate (tripalmitin purity $\geq 99 \%$ ), 1.2-dipalmitoyl-rac-glycero-3-phosphocholine (DPPC, 99\%), cholesterol ( $\geq 99 \%$ ), sodium azide, acetone (99.5\%), ethanol (99.8\%), chloroform ( $\geq 99 \%)$, antibiotic-antimycotic solution, papain, L-15 medium (Leibovitz), poly-L-lysine, glutaraldehyde solution and propidium iodide were purchased from Sigma-Aldrich (Germany). Tween ${ }^{\circledR} 20$ and DMSO ( $\left.\geq 99.7 \%\right)$ were purchased from Fisher BioReagents (USA). B-27 supplement $(50 \times)$, Hibernate-A medium, Neurobasal-A medium and glutamax-I were purchased from Gibco/Invitrogen. 3-(4,5-Di-methyl-thiazol-2-yl)-5-(3-carboxy-methoxyphenyl)-2-(4-sulfo-phenyl)-2H-tetrazolium (MTS) assay (CellTiter96 AqueousOne Solution) was purchased from Promega (Southampton, UK). Phosphate-buffered saline (PBS; Multicell, Wisent), fetal bovine serum advanced, heat inactivated (FBS; Capricorn Scientific), DNAse I (Biomatik), Hoechst 33342, Blocker BSA in TBS (Cat \#37520), antibody diluent (Cat\#003118) were purchased from Thermo Fisher Scientific (USA). Primary antibodies, including anti-Wnt3a (Cat \#ab28472) and anti-mTOR (Cat \#ab87540), and goat anti-mouse IgG Alexa Fluor 594 (Cat \#ab150116) and goat anti-rabbit IgG Alexa Fluor 633 secondary antibodies were purchased from Abcam (Cambridge, UK). Anti-Bcl-2 (Cat \#sc7382) was purchased from Santa Cruz Biotechnology (USA). Anticleaved-caspase-3 monoclonal antibody (Cat \#9664) was purchased from Cell Signaling Technology (USA). 


\section{Production of CurcuEmulsomes}

CurcuEmulsome was produced applying the conventional rotary evaporation procedure described by Bolat et al. ${ }^{52}$ In brief, lipids including tripalmitin $(80 \mathrm{mg})$, dipalmitoyl phosphatidylcholine $(8 \mathrm{mg}$ ) and cholesterol (2 $\mathrm{mg})$, together with curcumin $(16 \mathrm{mg})$, were first dissolved in an organic solvent, ie chloroform $(2 \mathrm{~mL})$. The solvent was completely removed in a rotary evaporator at $40^{\circ} \mathrm{C}$ and under 474 mbar. A dry lipid film was established on the bottom of the rotary flask. Double-distilled water was added to the flask and the system was set to rotation at $80^{\circ} \mathrm{C}$ under atmospheric pressure for 4 hours to obtain emulsomes. To homogenize the particle size, emulsomes were applied to an ultrasonication bath at $70^{\circ} \mathrm{C}$ for 1 hour. To spin down unincorporated curcumin within the solution, preparations were centrifuged at $13,200 \mathrm{rpm}(16,100 \mathrm{~g})$ and $4^{\circ} \mathrm{C}$ for 10 minutes. The supernatant including the CurcuEmulsomes was stored at $4^{\circ} \mathrm{C}$ until required. Blank emulsomes were prepared following the same procedure without the addition of curcumin.

\section{Physicochemical Analysis}

The mean particle size and zeta potential of emulsomes were determined by dynamic light scattering using a Zetasizer instrument (Nano ZS; Malvern Instruments, UK). Accordingly, samples were diluted in $1 \mathrm{mM} \mathrm{KCl}$ solution to suitable concentrations. All analyses were performed in the auto-measuring mode at $25^{\circ} \mathrm{C}$. The average of triplicate analyses was recorded for each sample.

\section{Stability}

Particular stability of the formulations was assessed based on changes in size, zeta potential and polydispersity in time. The average size, zeta potential and polydispersity index (PDI) of the samples were analyzed periodically up to 11 months using a Zetasizer instrument (Nano ZS; Malvern Instruments, UK).

\section{Morphology: Particle Size and Shape}

The particular size of the emulsomes was further investigated with scanning electron microscopy (Zeiss EVO-HD -15 ; Germany) together with their shape and morphology. The pretreatment procedure comprised a short-term fixation where samples were placed on an aluminum holder and left at $4^{\circ} \mathrm{C}$ overnight for drying. Dried samples were then treated with PBS buffer containing 2.5\% glutaraldehyde for 15 minutes. Samples were washed three times with distilled water for 10 minutes. After gold sputtering
(EM ACE200; Leica, Germany), samples were investigated under the electron microscope.

\section{Quantification of Curcumin Inside CurcuEmulsomes}

The concentration of curcumin encapsulated inside CurcuEmulsomes was estimated as described elsewhere. ${ }^{51,52}$ A $1 \mathrm{mg} / \mathrm{mL}$ stock solution of curcumin was prepared in DMSO. A standard curve, generated by successive dilution of the stock solution $(5,10,20,50,100 \mu \mathrm{g} / \mathrm{mL})$ in a 96-well microplate (NEST Scientific, Cat \#701001, China), was used to determine curcumin concentrations in samples prepared by 1:10 dilution of CurcuEmulsome suspension in DMSO. Sample absorbance was measured at $430 \mathrm{~nm}$ on a UV-vis spectrophotometer (Spectramax i3 Multi-Mode Microplate Reader Detection Platform; Molecular Devices, Sunnyvale, CA, USA). A standard curve was prepared from the values of standards. The curcumin concentration of CurcuEmulsomes was estimated by the readout of the absorbance intensity and corresponding concentration on the standard curve.

\section{Encapsulation Efficiency}

The encapsulation efficiency of CurcuEmulsomes was calculated according to the following equation:

$$
\begin{aligned}
\text { Encapsulation Efficiency }(\%)= & \frac{W_{\text {incorporated compound }}}{W_{\text {total }}} \\
& \times 100 \%
\end{aligned}
$$

where $W_{\text {incorporated compound }}$ is the amount of curcumin in the emulsomes; and $W_{\text {total }}$ is the amount of curcumin used in the formulation.

\section{In Vitro Drug Release Analysis}

The direct dispersion method described by Bisht et al and Anitha et al was applied for the in vitro drug release profile of the CurcuEmulsomes, as described elsewhere. ${ }^{53,54}$ In brief, $4 \mathrm{~mL}$ of CurcuEmulsome solution was divided into 10 microcentrifuge tubes ( $400 \mu \mathrm{L}$ each) and kept in a thermostable shaker at $37^{\circ} \mathrm{C}$ and $300 \mathrm{rpm}$. At time zero and at each time interval $(1 / 2,1,2,3,6,12,24$, 48, 72 hours), three tubes were taken and centrifuged at $3000 \mathrm{~g}$ for 10 minutes. The supernatant containing the emulsomes was removed. The pellet containing the released curcumin was dissolved in $400 \mu \mathrm{L}$ DMSO. The protocol described in the previous section was followed to quantify the amount of curcumin corresponding to the amount of curcumin released from CurcuEmulsomes. The procedure was repeated three times. 


\section{Isolation of Primary Hippocampal Neurons}

The BALB/c mice on postnatal day 0 (P0) were euthanized by decapitation and the brains were removed. Hippocampi were dissected out, transferred into $1 \mathrm{~mL}$ of L-15 medium (Leibovitz; Sigma) containing 1\% antibiotic-antimycotic (penicillin-streptomycin) (Sigma), 1\% glutamax (Gibco), 2\% B-27 (Gibco) and $20 \mu \mathrm{L}$ of Papain (Sigma), and then agitated for 45 minutes at $4^{\circ} \mathrm{C}$. After the addition of DNAse, the cell suspension was triturated with the help of a fire-polished glass pipette. To stop the enzymatic activity, homogeneous solution was taken into the L-15 medium (Leibovitz; Sigma) containing $1 \%$ antibiotic-antimycotic (penicillin-streptomycin) (Sigma), 1\% glutamax (Gibco) and 2\% B-27 (Gibco) and $10 \%$ of fetal bovine serum, and was then incubated at room temperature for 10 minutes. To spin down the cells, the solution was centrifuged at $1200 \mathrm{rpm}$ for 5 minutes. The supernatant was removed and $1 \mathrm{~mL}$ of Neurobasal-A (NBA) medium containing 1\% antibioticantimycotic (penicillin-streptomycin) (Sigma), 1\% glutamax (Gibco) and 2\% B-27 (Gibco) was directly added to the cells in the pellet. Isolated hippocampal neurons were seeded into 96-well plates at a density of 20,000 cells per well, and into the 12-well plates and the $35-\mathrm{mm}$ cell culture dishes at 100,000 cells per well for each experiment. Hippocampal neurons were cultured at $37^{\circ} \mathrm{C}$ and $5 \%$ $\mathrm{CO}_{2}$ for 2 days to allow the axon growth to reach to a certain length.

\section{Treatment of Neurons with Curcumin and CurcuEmulsomes}

After 2 days in culture, primary hippocampal neurons showed optimum neurite outgrowth. Cells supplemented with $1 \mathrm{~mL}$ of NBA medium containing $1 \%$ antibioticantimycotic (penicillin-streptomycin) (Sigma), 1\% glutamax (Gibco) and 2\% B-27 (Gibco) were grouped for the MTS assay as follows: (1) control group including the untreated cells, (2) vehicle control group including 0.01\% DMSO, (3) vehicle control group including blank emulsomes, (4) $2 \mu \mathrm{M}$ free curcumin (ie curcumin dissolved in DMSO), (5) $5 \mu \mathrm{M}$ free curcumin, (6) $10 \mu \mathrm{M}$ free curcumin, (7) $2 \mu \mathrm{M}$ CurcuEmulsome, (8) $5 \mu \mathrm{M}$ CurcuEmulsome, and (9) $10 \mu \mathrm{M}$ CurcuEmulsome. The DMSO content in the total cell medium of free curcumin treatment groups was kept below $0.01 \%$ to avoid any influence of DMSO on the neurons. The vehicle control group (2) had a $0.01 \%$ DMSO content to monitor the influence of DMSO on neuron culture. For the succeeding axotomy and ICC studies, the experimental groups were kept limited, with (1) control group (untreated cells), (2) 5 $\mu \mathrm{M}$ free curcumin treatment group, and (3) $5 \mu \mathrm{M}$ CurcuEmulsome treatment group.

\section{In Vitro Cellular Uptake}

Following the treatment of neurons with curcumin and CurcuEmulsome, the neurons were investigated under a confocal laser scanning microscope (CLSM) periodically at 24,48 and 72 hours. Uptake of curcumin and CurcuEmulsomes by the neurons was tracked and cell morphology was studied for the presence of any alterations. Accordingly, at DIV2 (day in vitro 2) following the cell seeding, cells were treated with $5 \mu \mathrm{M}$ free curcumin (curcumin dissolved in DMSO) and $5 \mu \mathrm{M}$ CurcuEmulsomes. Curcumin and CurcuEmulsome uptake was visualized with the help of the autofluorescent property of curcumin for 72 hours.

Cellular uptake was further studied by threedimensional (3D) Z-stack imaging via the addition of fluorescent agents at DIV2 after cell seeding. Red Vybrant Tongue (V22885; Thermo Fisher Scientific Orange Red) was used to stain entire cells and Hoechst 33342 (H3570; Thermo Fisher Scientific) (3:1000) was used to stain nuclei. Following 10 minutes of staining, CurcuEmulsomes were added to the culture and images were taken in series for 15 minutes from different Z-stacks with $1-\mu \mathrm{m}$ intervals. When the imaging was complete, images were merged to realize the $3 \mathrm{D}$ structure using the ZEN program (Zeiss).

\section{MTS Assay}

A commercially available one-solution cell proliferation assay containing a novel tetrazolium compound (MTS [3-(4,5-dimethylthiazol-2-yl)-5-(3-carboxymethoxyphenyl)-2-(4-sulfophenyl)-2H-tetrazolium]) and an electron coupling reagent (phenazine ethosulfate; PES) (CellTiter $96^{\circledR}$ Aqueous, Promega) was used to test the effects of free curcumin and CurcuEmulsomes on the viability of primary hippocampal neurons. NADPH or NADH produced by metabolically active cells causes reduction of the MTS tetrazolium compound (Owen's reagent) into a colored formazan product that can be detected at 490 $\mathrm{nm}$. At certain time intervals (DIV1, DIV2 and DIV3) after the treatments, the culture medium was discarded and the cells were washed with PBS. Then, $100 \mu \mathrm{L}$ NBA medium (minus phenol red and without additives) containing $20 \mu \mathrm{L}$ of the MTS solution was added to the 
culture. Cells were incubated for 3 hours at $37^{\circ} \mathrm{C}$ and the absorbance of formazan was read at $490 \mathrm{~nm}$ using a microplate reader (Spectramax i3 Multi-Mode Microplate Reader Detection Platform; Molecular Devices, Sunnyvale, CA, USA).

\section{Formation of Laser Beam-Induced Injury: Axotomy}

To make it easier to track the injured neurons throughout the experiment, a gridline pattern was plotted on the surface of the dishes prior to seeding the cells. A laser microdissection system (PALM CombiSystem; Zeiss), operating with a 355-nm UV laser (1-100 pulse production per second with an energy release equivalent to $90 \mu \mathrm{J}$ ), was used for the induction of neurite injury. Neurons that had no bleb, vacuoles, beaded axons or damaged membrane on their surface were selected and marked using ZEN software. The axotomy procedure was carried on PALMRobo software using a 40x dry phase contrast objective. The laser was applied to the selected neurites, each time with a distance of 25-30 $\mu \mathrm{m}$ from the neuronal body.

Axotomy was applied after the neurons (DIV2) had been exposed to curcumin or CurcuEmulsome treatment for 6 hours. The axotomy study involved six different experimental groups (three test groups and three control groups), which were thereafter assessed through both cell viability and immunocytochemistry analysis:

1. Untreated control group: no treatment, no injury.

2. Sham control group: the laser focus was adjusted to an empty spot 25-30 $\mu \mathrm{m}$ from the neurons to determine whether the laser causes any effect on cell viability or degeneration via heating or radiation (applied only at cell viability analysis).

3. Untreated injured group: no treatment was applied to this group following axotomy, to investigate the effect of injury when the cells are not treated.

4. Curcumin-treated control group: neurons (DIV2) were treated with $5 \mu \mathrm{M}$ curcumin; no axotomy was applied (for immunocytochemistry analysis only).

5. Curcumin-treated injured group: neurons (DIV2) were treated with $5 \mu \mathrm{M}$ curcumin; axotomy was applied 6 hours after treatment.

6. CurcuEmulsome-treated injured group: neurons (DIV2) were treated with $5 \mu \mathrm{M}$ CurcuEmulsomes; axotomy was applied 6 hours after treatment.

The axotomy procedure involved axotomy on 50 neurites for each petri dish, and was repeated four times for both cell viability and immunocytochemistry, making a total of 200 neuron injuries for each study group.

\section{Cell Viability After Injury}

Cell survival upon neurite injury was assessed by propidium iodide (PI) and Hoechst 33342 staining 24 hours after the axotomy procedure. A PALM microdissection microscope (Zeiss, Turkey) equipped with an integrated stage-top incubator regulated the cell environment at $37^{\circ} \mathrm{C}$ and $5 \% \mathrm{CO}_{2}$ throughout this experiment. Tile images were taken using a $20 \times$ lens as the gridline patterns on the surface of the culture dishes were adjusted in the center of the screen. For each of the six experimental groups, 50 healthy neurons were selected and marked for the axotomy. Twenty-four hours after the laser-induced neurite injury, the medium was replaced with fresh medium containing Hoechst 33342 (1:1000). Cells were incubated again at $37^{\circ} \mathrm{C}$ and $5 \% \mathrm{CO}_{2}$ for a further 10 minutes. Then, PI $(1: 1000)$ was added to the medium and cells were incubated under the same conditions for an additional 5 minutes. Fluorescent images of the marked locations were acquired on a PALM microdissection microscope. Fluorescent signals of Hoechst and PI were detected at wavelengths of 350/461 nm and 535/617 nm (excitation/emission), respectively, with a $20 \times$ objective. Viable (Hoechst-positive; PI-negative) and dead neurons (Hoechst-positive; PI-positive) were manually counted using ImageJ software.

\section{Immunocytochemistry}

Twenty-four hours after the laser-induced neurite injury, primary hippocampal neurons were fixed by incubation in 4\% paraformaldehyde in PBS for 15 minutes at room temperature. Cells were then washed twice with PBS and incubated in a blocker solution (Blocker BSA in TBS; Thermo Fisher Scientific) in PBS for 90 minutes. Thereafter, the blocking solution was discarded and neurons were washed twice with PBS at room temperature. Subsequently, neurons were treated with the primary antibodies with different dilution ratios for 4 hours at room temperature as follows: rabbit polyclonal anti-Wnt3a antibody [1:200] (Abcam ab28472), mouse monoclonal [53E11] to anti-mTOR antibody [1:200] (Abcam ab87540), mouse monoclonal IgG $_{1}$ anti-Bcl-2 antibody [1:200] (Santa Cruz Biotechnology, sc7382) and rabbit monoclonal caspase-3 (Cell Signaling Technology, 8G10) diluted in antibody diluent (Thermo Fisher Scientific). Cells were washed with PBS and incubated at room temperature with 1:200 diluted goat anti-mouse IgG Alexa Fluor 594 or goat anti-rabbit IgG Alexa Fluor 633 secondary 
antibodies for 90 minutes. Cells were washed three times with PBS containing $0.2 \%$ Tween-20 for a total of 12 minutes. Cells were then incubated in DAPI solution [1:1000] diluted in PBS for a further 3 minutes at room temperature. Finally, cells were washed with PBS once and incubated in PBS containing $0.1 \%$ sodium azide. The marked neurons on the gridline pattern were positioned using a $10 \times$ objective lens. After localization of the injured neurons, images were captured with a $40 \times$ oil-immersion lens using a confocal microscope (LSM 800; Zeiss). DAPI was detected at wavelengths of 358/461 nm, Alexa Fluor 594 at 590/617 nm and Alexa Fluor 633 at 632/642 nm (excitation/emission).

\section{Image Analysis Using Image J}

Analysis of the total dead-alive cells, total fluorescence per cell and corrected integrated density for each cell was performed using the image analysis platform Fiji (ImageJ). ${ }^{55}$

\section{Statistical Analyses}

Values were calculated using GraphPad Prism Software (version 6.01) and presented as mean \pm standard error of the mean (mean \pm SEM). Statistical analyses were performed using one-way ANOVA post hoc Tukey's test for the viability and ICC analysis. The chi-squared test was performed to assess the viability following the neurite injury. The level of significance was set at $P<0.05$.

\section{Results}

\section{Characterization Studies \\ Dispersity}

Free curcumin is poorly soluble in aqueous media, with macroscopic undissolved flakes of the compound visible in particular on the surface of the solution (Figure 1A), whereas CurcuEmulsome is a clear, dispersed formulation, with its hue derived from the natural color of curcumin. The dispersity was observed to be stable for at least 11 months under storage conditions of $4^{\circ} \mathrm{C}$.

\section{Physicochemical Properties of CurcuEmulsomes}

DLS analysis with a Zetasizer Nano ZS (Malvern Instruments, UK) determined the average diameter of 10 distinct CurcuEmulsome formulations as $178.8 \pm 13.7 \mathrm{~nm}$ (with an average polydispersity index of $0.18 \pm 0.06$ ) (Table 1), where the plus-minus sign indicates the margin of average sizes of numerous CurcuEmulsome productions with the same composition. A size distribution curve indicated a variation in particle size in the range of $75-450 \mathrm{~nm}$ (Figure 2A; Supplementary Material, Table S.1). Similarly, the average diameter of blank emulsomes was found to be $204.3 \pm 20.0 \mathrm{~nm}$, with an average PDI value of $0.269 \pm 0.050$. With a moderate PDI value (between 0.01 and 0.04 ), both emulsomes and CurcuEmulsomes displayed slightly polydisperse characteristics (Table 1). In addition, loaded with negatively charged curcumin, CurcuEmulsomes differed from blank emulsomes $(-24.2 \pm 6.3 \mathrm{mV})$, with a higher negative average zeta potential $(-33.2 \pm 6.7 \mathrm{mV})$ (Figure 2B).

\section{Stability}

Protected from light and stored at $4{ }^{\circ} \mathrm{C}$, both emulsomes and CurcuEmulsomes conserved their average size, zeta potential and PDI values for as long as 11 months (Table 2). The conserved physicochemical characteristics indicated no occurrence of significant loss of structural integrity of the formulations.

\section{Morphology}

Scanning electron micrographs verified that CurcuEmulsomes are spherical in shape, and display smooth, uniform surface characteristics in morphology (Figure 1B). In parallel with the DLS data, the size of CurcuEmulsomes was seen to be around $200 \mathrm{~nm}$ on average, with a particle size distribution between 50 and $400 \mathrm{~nm}$.
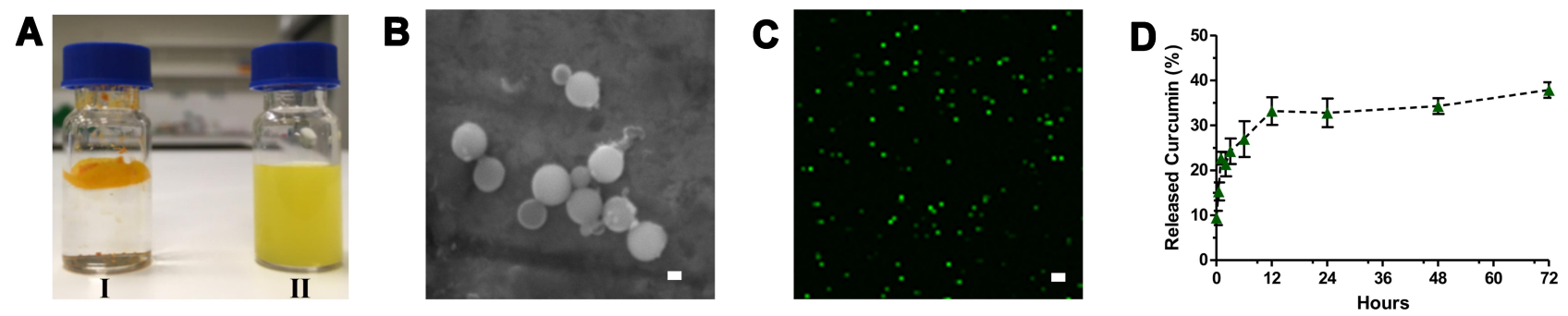

Figure I Characterization of CurcuEmulsomes. (A) Photographs of (I) curcumin, insoluble in water, and (II) CurcuEmulsomes, soluble in water. (B) Scanning electron micrograph of CurcuEmulsomes (scale bar $=200 \mathrm{~nm}$ ) (C) Confocal laser scanning microscopy (CLSM) image of CurcuEmulsomes in aqueous environment (scale bar=200 $\mathrm{nm}$ ). (D) In vitro drug release profile of CurcuEmulsomes during 72 hours. Values represent mean \pm SEM from three independent experiments. 
Table I ZetaSizer Analysis Data: Average Particle Size, Polydispersity Index (PDI) and Average Zeta Potential Values of All CurcuEmulsome and Emulsomes Produced and Used Throughout the Study

\begin{tabular}{|l|l|l|l|}
\hline Formulation & $\begin{array}{l}\text { Particle } \\
\text { Diameter } \\
(\mathbf{n m})\end{array}$ & PDI & $\begin{array}{l}\text { Zeta } \\
\text { Potential } \\
(\mathbf{m V})\end{array}$ \\
\hline $\begin{array}{l}\text { Emulsome } \\
\text { CurcuEmulsome }\end{array}$ & $204.3 \pm 20.0$ & $0.269 \pm 0.050$ & $-24.2 \pm 6.3$ \\
$176.8 \pm 13.0$ & $0.180 \pm 0.060$ & $-33.2 \pm 6.7$ \\
\hline
\end{tabular}

\section{Curcumin Encapsulation}

As estimated by absorbance measurements, CurcuEmulsomes achieved average curcumin encapsulation of around $0.08 \mathrm{mg} / \mathrm{mL}$ (in the range of $0.06-0.11 \mathrm{mg} / \mathrm{mL}$ ), where the maximum value corresponds to a 10,000 -fold increase in solubility of curcumin, as previously reported by Ucisik et al. ${ }^{51}$ CLSM analysis further provided evidence not only for the
Table 2 Stability Data of CurcuEmulsomes: ZetaSizer Analysis Data of CurcuEmulsomes on the Day of Production and at I Month, 2 Months, 4 Months and II Months After Production

\begin{tabular}{|l|l|l|l|}
\hline Time & $\begin{array}{l}\text { Average } \\
\text { Particle } \\
\text { Diameter }(\mathbf{n m})\end{array}$ & PDI & $\begin{array}{l}\text { Average Zeta } \\
\text { Potential } \\
(\mathbf{m V})\end{array}$ \\
\hline Day of production & 162.6 & 0.101 & $-28.0 \pm 7.5$ \\
2 months later & 164.4 & 0.096 & $-21.1 \pm 6.7$ \\
4 months later & 157.0 & 0.026 & $-25.8 \pm 5.0$ \\
II months later & 149.6 & 0.096 & $-25.0 \pm 7.3$ \\
\hline
\end{tabular}

presence of curcumin within each emulsome particle (Figure 1C), but also for the lack of any liposomes as by-product, which would be seen as hollow circles under a fluorescent microscope. In addition, CLSM images pointed out the highly dispersed character of CurcuEmulsomes in water. In the production of CurcuEmulsomes, curcumin was used in excess

\section{A}

Size Distribution by Intensity

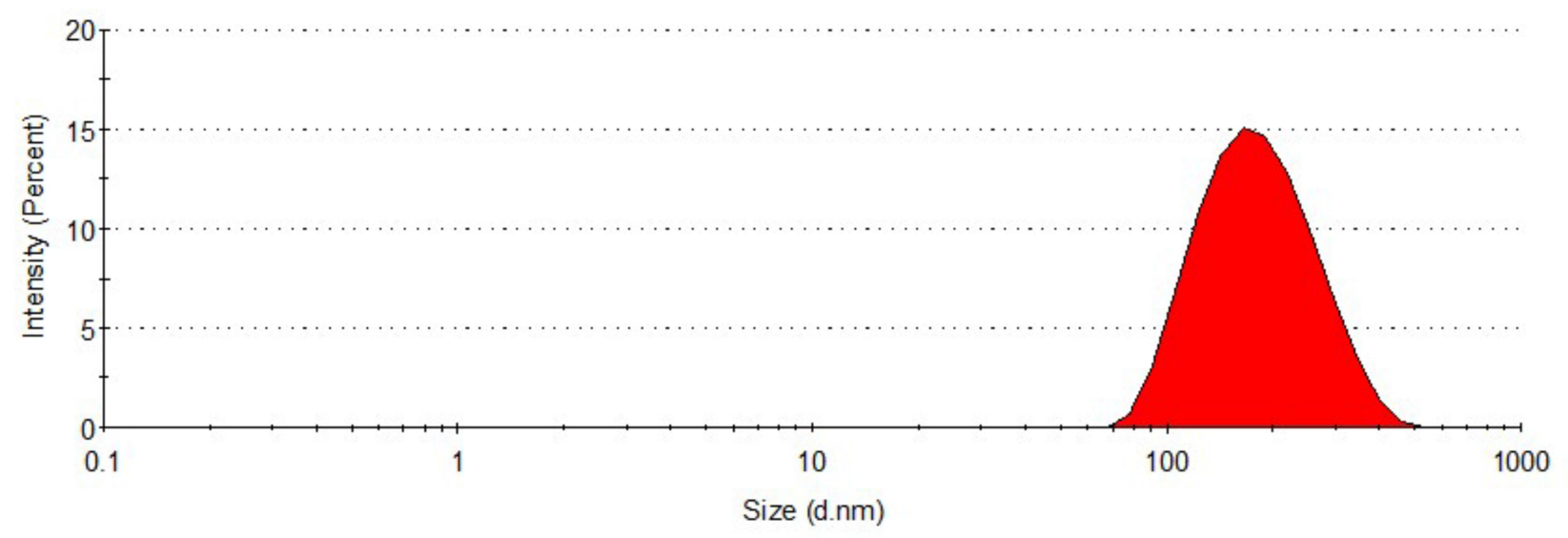

B

Zeta Potential Distribution

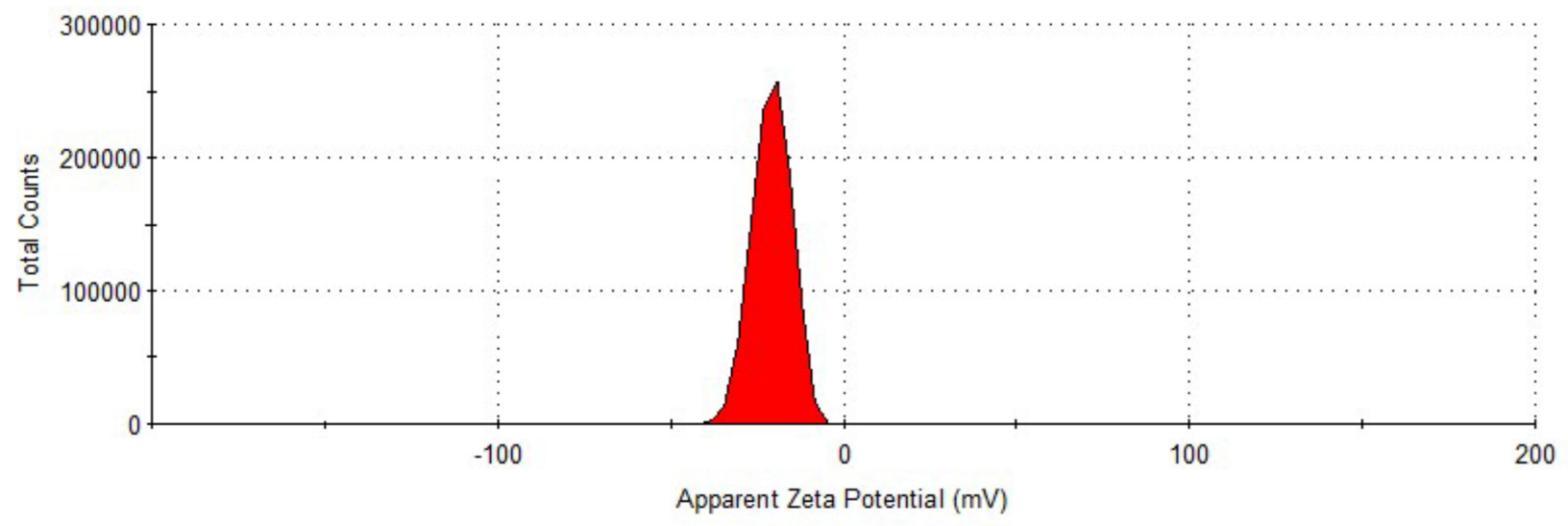

Figure 2 (A) Size distribution and (B) zeta potential curves of CurcuEmulsomes. 
compared to other lipid components to enable the maximum amount of encapsulation of the lipophilic compounds. The encapsulation efficiency of curcumin inside emulsomes was calculated as $4.8 \%$.

\section{In Vitro Drug Release}

The release profile suggested that CurcuEmulsomes release approximately $20 \%$ of their curcumin content within the first 6 hours in vitro, increasing to nearly $35 \%$ at 12 hours and, upon reaching a maximum, remaining constant (Figure 1D). Drug release data claim that no further release occurs between 12 and 72 hours, which is regarded as questionable. Owing to the low solubility and poor stability of curcumin in water, it is thought that the in vitro release data do not accurately reflect the actual curcumin release occurring from CurcuEmulsomes. This will be further elaborated in the discussion.

\section{Cell Culture Studies}

\section{Isolation of Primary Hippocampal Neurons}

Neurite growth of around $100 \mu \mathrm{m}$ was set as a requirement to proceed to laser axotomy. Upon the isolation of neurons at postnatal day 0 , neurite growth was observed for 3 days.
Two days of incubation was found to be sufficient for the required neurite elongation. Accordingly, depending on the time of day when the isolation process was carried out (ie in the morning or in the evening), neurite lengths were observed to become reproducibly equal to or greater than $100 \mu \mathrm{m}$ at 2 and 3 days after the neuron seeding (Figure 3A).

\section{Cell Uptake Studies of Curcumin and CurcuEmulsomes}

The autofluorescence property of curcumin enabled monitoring of CurcuEmulsome uptake by the primary hippocampal neurons using CLSM. Three-dimensional Z-stack imaging provided evidence that the uptake mechanism works quickly for both free curcumin and CurcuEmulsomes. Within 15 minutes, fluorescent signal of the compound could be detected largely not only in the membrane, but also inside the neuron body and the cytoplasm (Figure 3B). Merged images of membrane stain DiI (red) and curcumin (green) demonstrate that fluorescent signals of both curcumin and DiI are overlapping throughout the cell body (Figure 3B; Additional file 1: Video S1). This indicates once again that curcumin, with its lipophilic

B
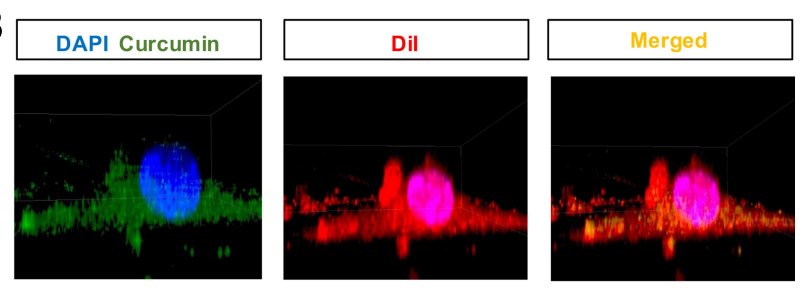

D

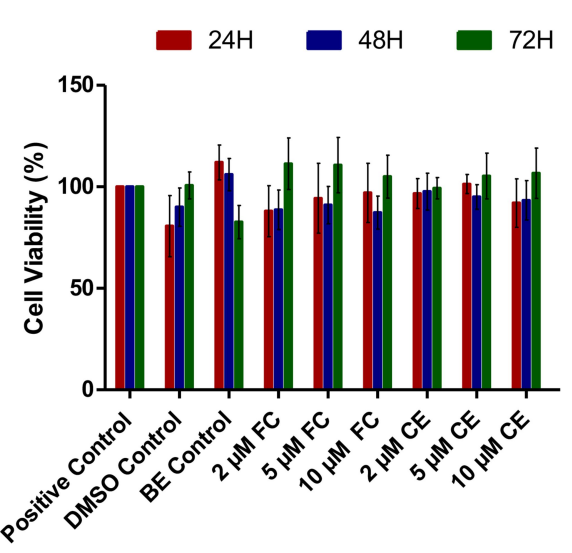

Figure 3 Primary hippocampal neuron culture: cellular uptake and safety of curcumin and CurcuEmulsomes. (A) Microscopy images of primary hippocampal neuron culture showing the growth of the neurites from day I to day 3 after cell seeding (scale bars $=20 \mu \mathrm{m}$ ). (B) 3D Z-stack CLSM images of primary hippocampal neurons showing the cell uptake of CurcuEmulsomes during the first 15 minutes of the treatment: curcumin inside CurcuEmulsomes (green), nucleus stained with DAPI (blue), membrane stained with Dil (red). (C) CLSM images of curcumin- and CurcuEmulsome-treated (5 $\mu$ M) hippocampal neurons from DIV0 to DIV3 (scale bars=20 $\mu$ m). (D) Safety of CurcuEmulsomes: cell viability data of primary hippocampal neurons treated with various curcumin and CurcuEmulsome concentrations (ie 2,5 and $10 \mu \mathrm{M}$ ) at 24,48 and 72 hours. Values are reported as untreated control normalized to 100\%. Values represent mean+SEM from four independent MTS analyses.

Notes: There were no statistically significant differences between group means as determined by one-way ANOVA post hoc Tukey analysis, $P>0.05$.

Abbreviations: BE, blank emulsome; FC, free curcumin; CE, CurcuEmulsome. 
character, largely prefers localization within the lipophilic compartments inside the cell, as the DiI membrane stain does. Moreover, no overlap occurs between the signals of DAPI (blue) and curcumin (green), indicating that CurcuEmulsomes do not enter the nucleus. Although this study is the first to apply emulsomes as the nanocarrier system to the primary hippocampal neurons, inaccessibility of the system to the nucleus is not unexpected. A previous study on the HepG2 carcinoma cell line observed CurcuEmulsomes in the membrane and in the cytoplasm, but not inside the nucleus. ${ }^{51}$ Free curcumin was observed not to localize inside the nucleus (Figure 3B and C).

Uptake and localization of free curcumin and CurcuEmulsomes in primary hippocampal neurons were further investigated with confocal microscopy for 72 hours after treatment. Upon cell uptake, compartmental localization of both free curcumin and CurcuEmulsomes inside the neuron was further investigated 72 hours after treatment. Delivered as a free agent or within the emuslome, curcumin was found to accumulate at the neuronal body and along the axons (Figure 3C). Neurons treated with free curcumin treatment $(5 \mu \mathrm{M})$ were distinguished from neurons treated with CurcuEmulsomes $(5 \mu \mathrm{M})$ at DIV1 by a brighter fluorescent signal, indicating the higher curcumin uptake into the neuron. At DIV2, indicating a similar or equal curcumin uptake, the fluorescence intensities of both neuron groups became comparable and the neurons appeared very bright, with the curcumin present inside. At DIV3, fluorescence signals weakened for both curcuminand CurcuEmulsome-treated neurons, yet highly fluorescent spherical regions were detected inside the neurons treated with CurcuEmulsomes. In previous studies, the cellular uptake mechanism of emulsomes was discovered to be endocytosis. ${ }^{51,56}$ The inset image in Figure $3 \mathrm{C}$ indicates a similar observation that upon uptake, CurcuEmulsomes localize inside the endosomes within the cell and sustain curcumin release from the inside of the endosome with time. Therefore, the punctual bright spots inside the inset image are attributed to the endosomes carrying the CurcuEmulsomes.

\section{MTS Assay}

MTS cell viability tests indicated that neither free curcumin nor CurcuEmulsomes cause any toxicity on primary hippocampal neuron culture within a concentration range of 2-10 $\mu \mathrm{M}$. No significant difference was observed between any treatment group and the untreated control $(P>0.05)$
(Figure 3D). In addition to the MTS cell viability assay, PI and Hoechst 33342 staining was applied to primary hippocampal neurons to evaluate the cell viability after treatment with varying curcumin and CurcuEmulsome concentrations (eg 2-10 $\mu \mathrm{M}$ ). Hoechst (blue) labeled viable cells, while PI (red) labeled dead cells (Supplementary Material, Figures S.1 and S.2). Supportively, no significant difference was observed between any treatment group and the untreated control $(P>0.05)$.

\section{Cellular Viability After Neurite Injury}

During laser axotomy, the laser was applied to the neurite at a distance of $25-30 \mu \mathrm{m}$ from the neuronal body, ensuring that a replicable neurite injury could be established. Microscopy images of neurites before and after the laser axotomy (black arrows) were visualized by a microscope (Figure 4A; Additional file 2: Video S2). Then, 24 hours after the injury, PI and Hoechst 33342 staining were applied to primary hippocampal neurons to quantify the survival rates of the injured neurons. Viable cells were labeled with Hoechst (blue), while dead cells were labeled with PI (red) (Supplementary Material, Figure S.3). Cell survival rates are quantitatively displayed as bar-graphs in Figure 4B. In the absence of axotomy, approximately $83 \%$ of primary hippocampal neuron culture was found to be alive. Likewise, the sham control group showed approximately $82 \%$ viability. This demonstrates that the laser beam had no effect on cell survival by means of heat or radiation when applied to the cell environment. Laser axotomy negatively affected cell viability and cell survival rate at approximately $52 \%, 24$ hours after the injury. When treated with $5 \mu \mathrm{M}$ free curcumin, $67 \%$ of the neurons remained alive after the injury. The difference in cell survival rates was statistically significant $(P<0.05)$. Similarly, CurcuEmulsome treatment with equal curcumin concentration (ie $5 \mu \mathrm{M}$ ) improved the cell survival rate to $69 \%$. This increase not only was significant compared with the survival rate of the injured control group, but also surpassed the value of free curcumin treatment.

\section{Immunocytochemistry Analysis}

Neurons immunostained with anti-Wnt3A, -Bcl2, -mTOR and -cleaved caspase 3 were further investigated under confocal fluorescence microscope to quantitatively study the expression levels of these biomarkers (Figure 5). Accordingly, the fluorescence intensities of 50 neurons were analyzed for each sample and the average expression levels were quantitatively defined for each study group. The 
A

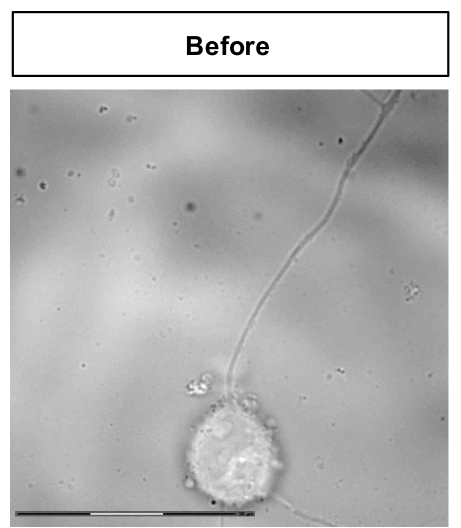

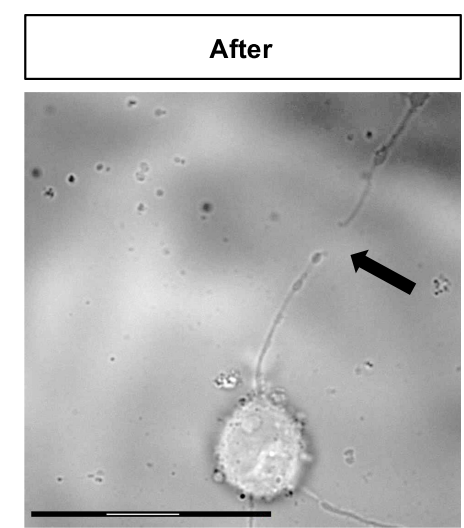

B

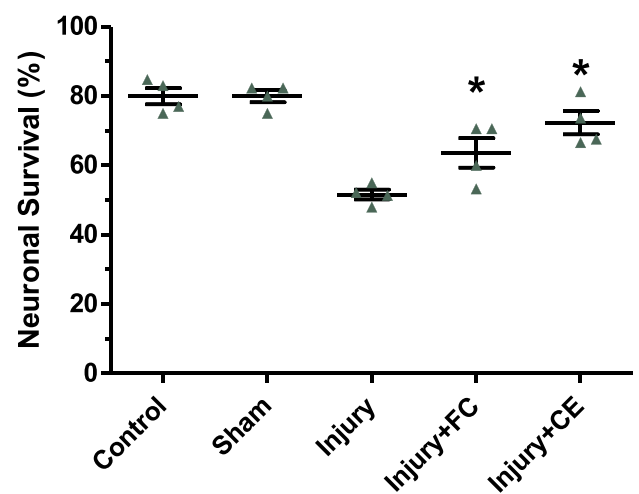

Figure 4 Laser axotomy of neurites. Microscopy images of a hippocampal neuron (A) before and (B) after axotomy was applied. The arrow indicates the point where axotomy was applied on a neurite of the single neuron (scale bars $=30 \mu \mathrm{m}$ ). (C) Survival rates of the neurons 24 hours after the axotomy injury. *Statistically significant difference from the axotomy group $(P<0.05)$.

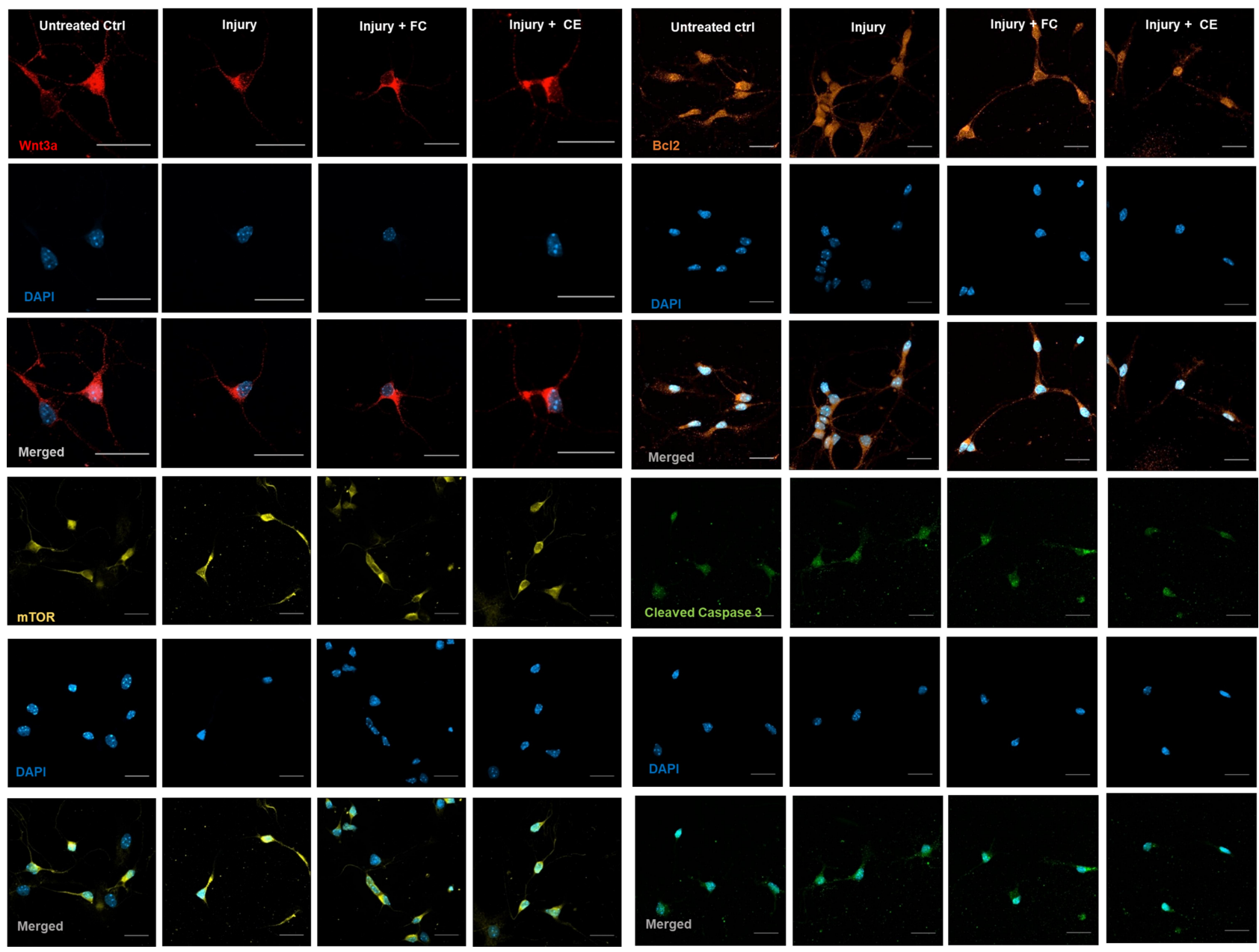

Figure 5 Microscopic immunocytochemistry (ICC) analysis. Representative CLSM images of Wnt3a, mTOR, Bcl 2 and cleaved caspase 3 markers of the treatment groups (scale bars $=20 \mu \mathrm{m}$ ).

Abbreviations: Ctrl, untreated control healthy neurons; Injury, only axon-injured neurons; FC+Injury, curcumin-treated injured neurons; CE+Injury, CurcuEmulsometreated injured neurons. 
control healthy neurons (ctrl), control injury group (injury), curcumin-treated healthy neurons (FC), curcumin-treated injury group (FC+injury) and CurcuEmulsome-treated injury group (CE+injury) were studied for expression levels of selected biomarkers (Figure 6). Since it is documented that unloaded lipid-based nanocarrier systems, with similar composition and physicochemical characteristics, have no effect on the CNS, ${ }^{57-62}$ blank emulsomes were not tested in axotomy studies.

The protein Wnt3a is essential for the regulation and maintenance of hippocampal neurogenesis. ${ }^{33}$ Significantly higher Wnt3a expression was observed in healthy cells when treated with curcumin, compared to both non- treated healthy and injured control groups (Figure 6A) However, after axotomy was applied, the increase in Wnt3a expression of curcumin-treated neurons was markedly reduced compared to the injury control. In contrast, CurcuEmulsomes did not cause any significant change in the level of Wnt3a expression of neurons after injury.

Mouse monoclonal anti-mTOR antibody was used to assess the expression of the mammalian target of rapamycin (mTOR) protein, a serine/threonine kinase. It has been previously shown that mTor participates in several processes in the brain, such as neural development of axons and dendrites. ${ }^{63}$ In hippocampal neurons, mTOR proteins were observed to exist largely within the cytoplasm of the
A
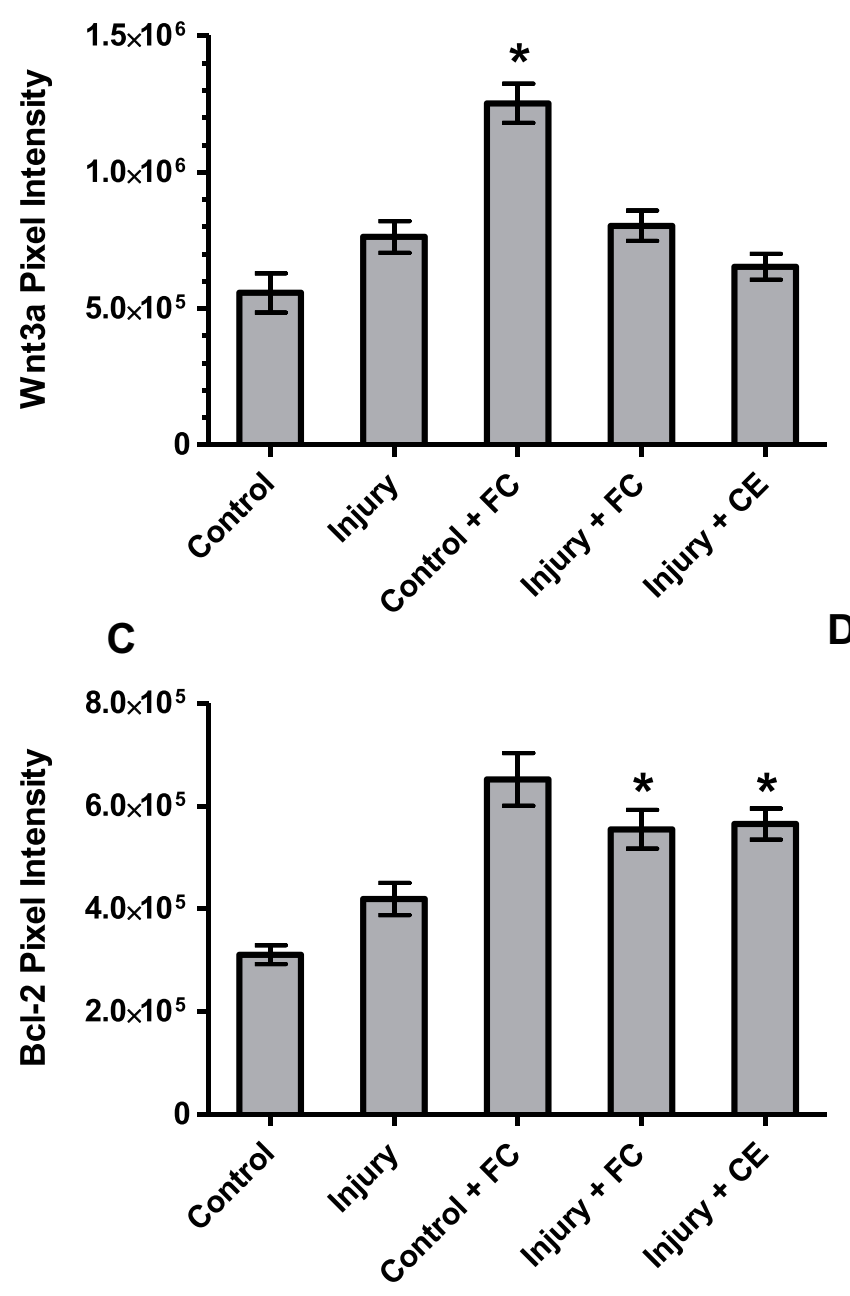

B
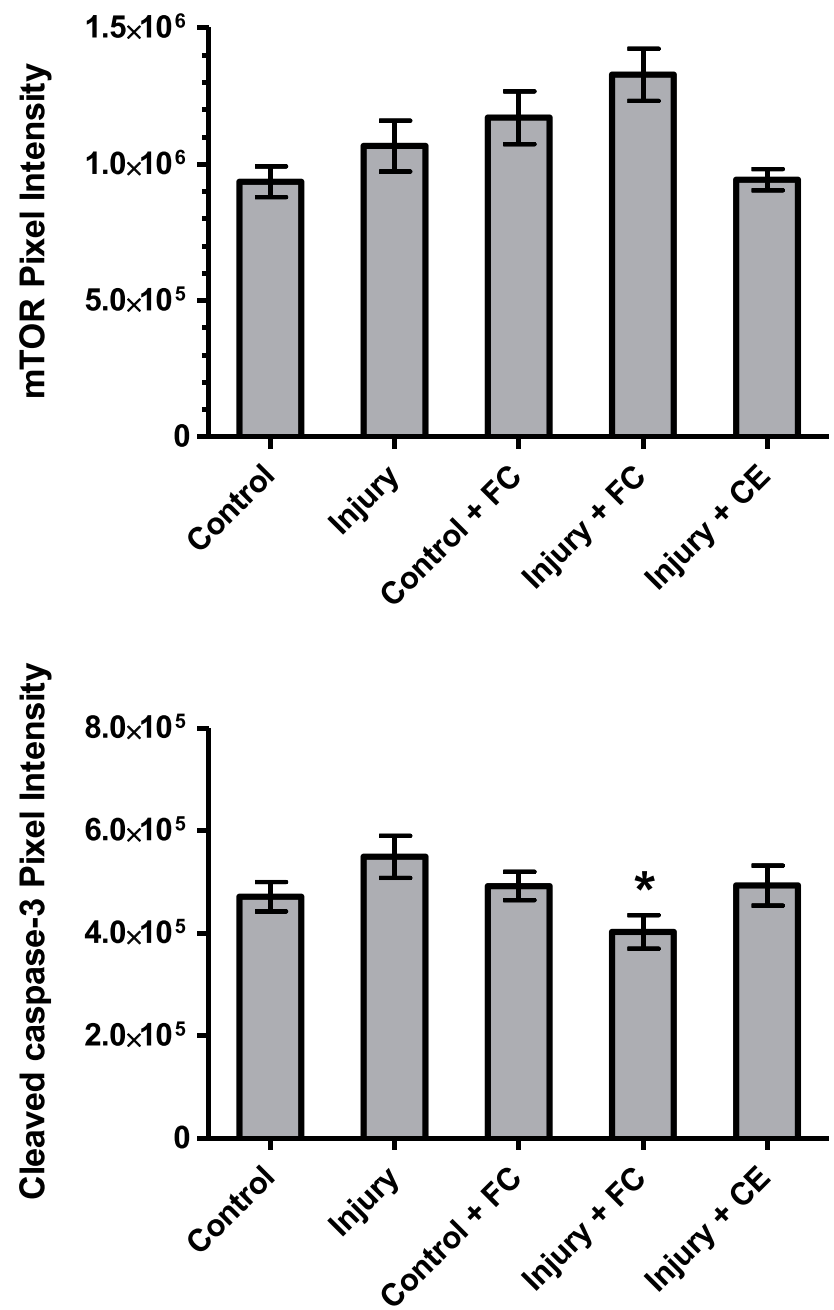

Figure 6 Quantitative ICC analysis. Integrated pixel intensity analyses of (A) Wnt3a, (B) mTOR, (C) Bcl-2 and (D) cleaved caspase-3 proteins. Values represent mean \pm SEM. *Statistically significant difference from the injury control group $(P<0.05)$.

Abbreviations: Ctrl, untreated healthy neurons; Injury, only axon-injured neurons; FC, curcumin-treated healthy neurons; FC+Injury, curcumin-treated injured neurons; CE +Injury, CurcuEmulsome-treated injured neurons. 
neuronal body and along the axons (Figure 5). As seen in Figure 6B, curcumin-treated injured neurons showed a slight increase in the expression of mTOR compared to the injured control group. CurcuEmulsome treatment did not induce any significant change in mTOR expression.

$\mathrm{Bcl}-2$ protein is a key regulator of endogenous apoptosis that also promotes neuronal survival in the $\mathrm{CNS} .{ }^{64} \mathrm{Bcl}-2$ was found to be localized particularly in the cytoplasm as well as within the nucleus, whereas its localization, and hence its expression level, was lower along the axons than in the cytoplasm and the nucleus (Figure 5). The quantification of fluorescence intensities revealed that both curcumin and CurcuEmulsome treatment groups had significantly higher Bcl-2 expression (Figure 6C) compared to the control injury group ( $P=0.011$ and 0.009 , respectively).

The cleaved caspase 3 apoptotic marker was observed to be present in the nucleus and cytoplasm (Figure 5). As reported previously, the translocation of cleaved caspase 3 from the cytoplasm into the nucleus may occur if apoptosis is induced. ${ }^{65}$ Accordingly, cleaved caspase 3 signal (green) was higher in the nucleus of the neurons than within the cytoplasm for all groups except the curcumintreated injury group (Figure 5). In the curcumin-treated injury group, the cleaved caspase 3 signal seen in the cytoplasm was more intense than the signal seen in the nucleus. Indeed, when the fluorescence intensities were compared, cleaved caspase 3 expression of curcumintreated injured neurons was found to be significantly lower $(P=0.026)$ compared to injured control neurons (Figure 6D). No significant difference was observed when the neurons were treated with CurcuEmulsomes after injury.

\section{Discussion}

\section{Characterization of CurcuEmulsomes}

While curcumin's low solubility (ie $11 \mathrm{ng} / \mathrm{mL}$ ) ${ }^{39}$ restricts further medicinal applications, nanocarrier systems hold great potential to address the bioavailability limitations. Composed of a solid fat core surrounded by a phospholipid layer, emulsomes offer an alternative approach to overcoming this limitation. Encapsulating curcumin inside a lipid matrix, CurcuEmulsomes could enhance the solubility of curcumin up to 10,000 fold, corresponding to $0.11 \mathrm{mg}$ curcumin per milliliter, as reported previously. ${ }^{51}$ CurcuEmulsome formulations produced for this study had an average concentration of 0.08 $\pm 0.02 \mathrm{mg} / \mathrm{mL}$. Scanning electron microscopy images presented the smooth morphology of the outermost phospholipid surface, thereby indicating the resemblance of emulsomes to the surface features of liposomes (Figure 1B). Accordingly, CurcuEmulsomes are round-shaped and have sizes in the range of $100-400 \mathrm{~nm}$. Size is predominantly determined by the phospholipid:tripalmitin ratio and can be further tuned through alteration of this ratio. ${ }^{56,66}$ Molecular physicochemical properties of lipid composition, as well as production and homogenization conditions (eg production/homogenization duration, temperature, mixing speed), are other factors that influence the final size of emulsomes. ${ }^{66}$ In addition, physicochemical properties of the drug incorporated into the emulsomes seem to have an effect on both the overall size and zeta potential of the formulation. ${ }^{52}$ For instance, CurcuEmulsomes can be distinguished from emulsomes with a higher negative charge $(-33.2 \pm 6.7 \mathrm{mV})$, which is attributed to the contribution of the molecular negative charge of curcumin to the formulation without curcumin $(-24.2 \pm 6.3 \mathrm{mV})$ (Table 1). Owing to a more negative zeta potential, CurcuEmulsomes seem to be distinguishable from blank emulsomes $(204.3 \pm 20.0 \mathrm{~nm})$, also with a smaller average particle size $(176.8 \pm 13.0 \mathrm{~nm})$, as reported elsewhere. ${ }^{52}$ The higher negative surface charge of CurcuEmulsomes is ascribed as the reason for their smaller average size in comparison to blank emulsomes. The polydispersity index data show a linearity between negative zeta potential and monodispersity (ie PDI values lower than 0.3 ) of the formulations (Table 1). Monodispersity of the formulation was further confirmed by confocal laser microscopy, as the autofluorescence of curcumin enabled the tracking of each particle (Figure 1C).

Average size, zeta potential and PDI values were traced by regular DLS analysis to investigate the long-term stability of the formulation. Mean particle diameter and zeta potential values remained steady (Table 2), indicating that CurcuEmulsomes preserve structural stability for at least 11 months. The solidity of the lipid core matrix, additionally supported and stabilized with a negatively charged phospholipid shell, is ascribed as the reason behind the long-term stability of the formulation. Nonetheless, despite the discovery that CurcuEmulsomes are structurally stable over a long period of time, and considering that release of even a small amount of curcumin from CurcuEmulsomes might influence the reproducibility of the experimental data, CurcuEmulsomes no older than 1 month were used in cell culture experiments throughout the study.

The drug release studies of CurcuEmulsomes demonstrate a prolonged release profile showing less than $40 \%$ curcumin release within the first 30 hours. This time point is particularly important in our study, as the viability and 
immunocytochemistry analyses were performed after 30 hours of CurcuEmulsome treatment, with axotomy being applied 6 hours after treatment and neurons analyzed 24 hours after axotomy. Surprisingly, the maximum curcumin release was achieved at around 35\% after 12 hours and the value remained stable until 72 hours (Figure 1D). The curcumin release profile of CurcuEmulsome resembles that of nanocurcumin described by Bisht et al, where $35 \%$ release was achieved after 12 hours. ${ }^{53}$ Similarly, Anitha et al reported burst release of curcumin in the first 6 hours followed by a gradual release, achieving around $40 \%$ release at 72 hours, with a total release as high as $65 \%$ after 120 hours. $^{54}$ Hence, the curcumin release profile of CurcuEmulsomes seems to display similar characteristics to two alternative delivery systems, ie polymeric $^{53}$ and dextran sulfate-chitosan nanoparticles, ${ }^{54}$ as reported previously. The incidence of a low release profile may be attributed as a result of two possible occurrences: First, the solid phase of the tripalmitin core of CurcuEmulsomes may result in a prolonged release of encapsulated curcumin at $37^{\circ} \mathrm{C}$, which is expected to occur. ${ }^{51}$ Second, the rapid denaturation of curcumin in the water ${ }^{67}$ may lead to underestimation of the amount of curcumin actually released. To express this more explicitly, the curcumin released from the CurcuEmulsomes may undergo denaturation repeatedly, so that the quantity of curcumin in the buffer solution detected each time may correspond to less than the actual release.

On the other hand, curcumin release within the first 3 hours was found to be around $20 \%$ of total curcumin content. Although this early, relatively high release percentage may seem contrary to the solid feature of the emulsome's solid core matrix, the reason is due to the characteristic structure of CurcuEmulsomes, as curcumin is located both within the hydrophobic regions of the phospholipid multilayer and inside the solid core matrix. ${ }^{66}$ Accordingly, curcumin loaded within the phospholipid layers of CurcuEmulsomes must have been released first. The relatively mobile nature of curcumin within the fluidic lipid matrix would allow its diffusion into the buffer more easily than the curcumin entrapped and immobilized within the solid fat core of CurcuEmulsomes (Figure 1D).

\section{Cell Studies: Cell Isolation, Uptake and Toxicity}

The isolation of mouse hippocampus was set at postnatal day 0 throughout the study. To contribute to the reproducibility of the data, a neurite growth of around 100 $\mu \mathrm{m}$ was set as a requirement to proceed to laser axotomy. Upon the isolation of neurons at postnatal day 0 , neurite growth was observed for 3 days, and 2 days of incubation was found to be optimum for neurite growth (Figure 3A).

As this study is the first report on emulsomes tested on primary hippocampal neurons, uptake of CurcuEmulsomes into the primary hippocampal neurons was examined in the first 15 minutes after treatment, to investigate the time taken for the delivery of curcumin into the neurons in vitro. CurcuEmulsomes were visible on the membrane and throughout the cytoplasm (Figure 3B). Cell uptake analysis showed that CurcuEmulsomes can enter the primary hippocampal neurons within 15 minutes and localize rapidly within the membrane and inside the cytoplasm. As this is the first study from our group reporting the use of emulsomes for delivery of neuroprotective agents into a primary neuron culture, it may be important to mention that the uptake of CurcuEmulsomes into the hippocampal neurons was observed to occur more rapidly compared to previously studied uptake rates of different emulsome formulations into cancer cells, such as HepG2, ${ }^{51}$ HCT116, ${ }^{52}$ LnCap (unpublished data) or MCF7 (unpublished data), in vitro.

Neurons were imaged each day during the 72-hour treatment with curcumin and CurcuEmulsomes. The mean fluorescence intensity of free curcumin was found to be at its highest on DIV1, while CurcuEmulsomes showed the brightest signal on DIV2 (Figure 3C) (quantitative data not shown). Spherical local spots with higher fluorescence intensities were observed inside the cytoplasm of the neurons treated with CurcuEmulsomes. The spots were interpreted as endosomes, as previous studies suggest that the uptake of emulsomes occurs through endocytosis..$^{51,56,68}$ The spots were not present in neurons treated with free curcumin, where uptake followed diffusion. ${ }^{69}$ Cell uptake studies verified that CurcuEmulsomes achieve delivery of curcumin into the hippocampal neurons, where curcumin mainly localized inside the membrane and in the cytoplasm. On the other hand, it is important to state that no curcumin signal was detected inside the nucleus throughout the 72 hour examination in either the free curcumin- or the CurcuEmulsome-treated groups.

Our study also revealed that curcumin is not toxic to primary hippocampal neurons at concentrations of 2, 5 and $10 \mu \mathrm{M}$ (Figure 3D). CurcuEmulsomes did not alter the viability of the neurons significantly when applied at the doses corresponding to the aforementioned curcumin 
concentrations, indicating the safety of the emulsome as the nanocarrier.

The effective dose of curcumin with a range of $250 \mathrm{nM}$ to $27 \mu \mathrm{M}$ has previously been reported, and is mainly dependent on the type of neuron culture studied, day of cell isolation and the cell origin. For instance, So et al reported that $500 \mathrm{nM}$ curcumin was the most effective in stimulating proliferation of neural progenitor cells (NPCs), whereas higher concentrations were cytotoxic. ${ }^{70}$ Ray et al reported that polymeric nanoparticle-encapsulated curcumin $\left(\mathrm{NanoCurc}^{\mathrm{TM}}\right)$ treatment significantly protects neuronally differentiated human SK-N-SH cells from $\operatorname{ROS}\left(\mathrm{H}_{2} \mathrm{O}_{2}\right)$ -mediated insults at doses between $250 \mathrm{nM}$ and $5 \mu \mathrm{M} .^{71} \mathrm{In}$ another study, high curcumin concentrations (ie 2.7-27 $\mu \mathrm{M}$ ) were found to be effective against $\mathrm{H}_{2} \mathrm{O}_{2}$-induced renal epithelial (LLC-PK1) cell injury with comparable inhibition of lipid peroxidation and lipid degradation. ${ }^{72}$ A study on primary cultures of cerebellar granule neurons (CGNs) demonstrated that curcumin $(10-15 \mu \mathrm{M})$ provides a neuroprotective effect against iodoacetate (IAA)-induced neuronal death. ${ }^{73}$ Srivastava et al proved the protective function of curcumin in arsenic-induced N-methylD-aspartate (NMDA) receptor dysfunction and PI3K/Akt/ GSK3b signaling on an in vitro primary hippocampal neuron model isolated from 6-8-day-old Wistar rats. ${ }^{74}$ In light of these findings, curcumin concentration was set as $5 \mu \mathrm{M}$ for the proceeding experiments, including laser axotomy. The safety data of CurcuEmulsomes (ie concentration $\leq 10$ $\mu \mathrm{M})$, as well as the observed particle density inside the neuron culture, had an influence on determination of the experimental concentration as $5 \mu \mathrm{M}$ for the axotomy and further studies.

\section{Injury and Immunostaining}

The previous studies on dorsal root ganglions (DRGs) reported an increase in neuronal death with increasing proximity of the injury to the cell body, attributed to the increased influx of extracellular $\mathrm{Ca}^{2+}$ generating a stronger injury current. ${ }^{75}$ This explanation for DRG cell death upon axotomy seems to be relevant also for the primary hippocampal model, where $52 \%$ of the injured neurons remained alive after induced injury at close proximity $(25-30 \mu \mathrm{M})$ to the cell body (ie data for the untreated injured group). Reducing injury, curcumin and CurcuEmulsome treatments significantly increased the survival rate of neurons to $67 \%$ and $69 \%$, respectively, compared to the untreated injured group ( $P=0.03$ and 0.01 , respectively) (Figure 4B). These findings provide evidence for the neuroprotective role of both curcumin and CurcuEmulsomes on hippocampal cells.

Wnts, lipid-modified secreted glycoproteins, signal through multiple pathways including the planar cell polarity, the $\mathrm{Wnt} / \mathrm{Ca}^{2+}$ and the canonical Wnt signaling pathway. The canonical Wnt signaling pathway regulates hippocampal neurogenesis, axonal remodeling and patterning. Among the members of the Wnt family, Wnt3a is essential for the regulation and maintenance of hippocampal neurogenesis. ${ }^{76}$ To date, various studies have shown that the use of neuroprotective agents results in upregulation of canonical Wnt3a signaling, and thus, further enhances neurogenesis. ${ }^{33,77-79}$ Following axonal injury, Wnt signaling could promote neuronal survival and regeneration of CNS axons of retinal ganglion cells. ${ }^{80}$ The neuroprotective effects of Wnt signaling include prevention of excitotoxicity and promotion of axonal growth in the adult spinal cord and sensory neurons following injury. ${ }^{80-85}$ In our study, curcumin treatment significantly upregulated Wnt3a on control cells, as expected $(P=0.00$ compared to untreated control cells). However, the observed increase in Wnt3a expression in injured cells upon treatment with curcumin or CurcuEmulsomes was not statistically significant (Figure 6A). Curcumin has previously been reported to activate the $\mathrm{Wnt} / \beta$-catenin pathway and increase Wnt3 expression and protein levels, leading to enhanced neurogenesis. ${ }^{26,33}$ It is important to emphasize that a significant increase in neurogenesis was observed at $0.5 \mu \mathrm{M}$ curcumin concentration, while higher doses $(\geq 5 \mu \mathrm{M})$ significantly reduced the proliferation/viability of hippocampal derived neural stem cells. ${ }^{33}$ This indicates that curcumin may display neuroregenerative effects in a concentration-dependent manner. These findings may be attributed to curcumin regulating multiple targets in different pathways. In our study, it is uncertain whether the insignificant increases in Wnt3a expression upon the curcumin and CurcuEmulsome treatment play a role in the improvement of survival rates of neurons after injury. However, the smaller increase in Wnt3a after CurcuEmulsome treatment - relative to curcumin treatment - may be attributed to the prolonged release profile of the nanocarrier system.

mTOR has a pivotal role in dendritic and axonal growth, synaptic plasticity and metabolic regulation. ${ }^{63}$ It has been reported that the mTOR signaling pathway has a crucial function in neuronal survival. ${ }^{86}$ As a pleiotropic molecule, curcumin affects numerous targets, including mTORC1 and mTORC2 signaling pathways in cancer 
cells, making curcumin a chemotherapeutic/chemopreventive agent against many types of cancer. ${ }^{87}$ According to our findings, curcumin treatment increased the level of mTOR expression compared to untreated control cells (Figure 6B). Curcumin-treated injured neurons exhibited the highest expression of mTOR among all groups, suggesting that curcumin has a significant role in the survival of injured neurons. In contrast, no significant alteration was observed in mTOR expression in CurcuEmulsometreated injured neurons. In other words, despite CurcuEmulsomes delivering curcumin into the cytoplasm successfully (Figure 3B and C), they did not cause the same effect on mTOR expression as curcumin itself. This may be attributed to the fact that CurcuEmulsomes are taken up by the neurons via endocytosis, followed by a slow but sustained release of curcumin from the endosome, as demonstrated by the release profile (Figure 1D). Ultimately, CurcuEmulsomes may not be enabling curcumin to achieve its effective concentrations within the cytoplasm in terms of mTOR expression.

Bcl2 is known as an anti-apoptotic gene. The members of the Bcl2 family are widely accepted as regulators of apoptosis. Furthermore, they also play a key role in normal cell physiology. ${ }^{88}$ It has been shown that Bcl 2 could block cell death in different cell types, including glia and neurons. ${ }^{89,90}$ Inhibition of the release of cytochrome $\mathrm{c}$ from mitochondria is a mechanism of action of $\mathrm{Bcl} 2$, which is particularly critical for the activation of caspases and, hence, apoptosis. ${ }^{90}$ It has previously been discovered that curcumin treatment increases the mRNA expression level of Bcl2. ${ }^{91}$ Likewise, in our study, curcumin treatment on untreated control neurons upregulated $\mathrm{Bcl} 2$ expression, with a modest difference between the free curcumin- and CurcuEmulsometreated axotomy-injured neurons $(P=0.453$ and 0.592 , respectively) (Figure 6C). Injured neurons treated with curcumin and CurcuEmulsome had a significant increase in the level of $\mathrm{Bcl} 2$ ( $P=0.011$ and 0.009 , respectively) compared to the injured control group. The findings of the Bcl2 immunostaining highlighted the anti-apoptotic activity of both curcumin and CurcuEmulsomes in the axotomy-injured hippocampal model.

Cleaved caspase 3 has been regarded as an apoptotic marker. ${ }^{92}$ Neurodegeneration and neuron death upon mechanical hazard are known to occur during and after induced apoptosis. ${ }^{93}$ In parallel, immunocytochemistry analysis revealed an increase in the expression of cleaved caspase 3 upon neuron injury. Counteracting the induction of apoptosis, curcumin significantly decreased cleaved caspase 3 expression compared to control cells (Figure 6D). CurcuEmulsome treatment also decreased the cleaved caspase expression level compared to the axotomy-injured group; however, the decrease remained limited compared to free curcumin treatment. This, as in the mTOR analysis, is again attributed to the sustained release profile of CurcuEmulsomes resulting in a lower available curcumin concentration and therefore activity inside the cytoplasm. These findings are consistent with the data from an ischemia-induced delayed neuronal death study, showing that inhibition of the mitochondrial-mediated apoptotic signaling cascade occurs through dietary supplementation with curcumin. ${ }^{94}$ Similarly, our findings suggest that curcumin treatment inhibited apoptotic signaling in axotomized cells. However, the anti-apoptotic effect of CurcuEmulsomes remained low compared to their free form applied at the same concentration, which is attributed to the slow release profile of the emulsomes.

Sustained release is widely accepted as advantageous in in vivo and clinical studies, offering less frequent dose administration and minimizing systemic side effects (as the drug is first released upon its uptake to the inflammatory organ or the tissue). In our in vitro example, however, where there was controlled release of curcumin to the system by CurcuEmulsomes, the neuroprotective effect remained lower than that for free curcumin at the same concentration. This is because the concentration of the curcumin released to the cytoplasm from the CurcuEmulsomes remained less than the total amount of curcumin encapsulated inside the cytoplasm for the period of the study. Higher concentrations of CurcuEmulsomes may be considered for future in vivo studies to improve the formulation's efficacy in neuroprotection against neural injury.

\section{Conclusion}

As verified for the first time on neurons, CurcuEmulsomes offer a prominent and safe approach to deliver curcumin in the axotomy-injured primary hippocampal cell model. Displaying stable, dispersed characteristics, CurcuEmulsomes achieved the delivery of curcumin to the hippocampal neurons. The formulation exhibited no toxicity against the primary hippocampal neuron culture in the tested range of $2-10 \mu \mathrm{M}$ curcumin. The neuroprotective activity of CurcuEmulsomes was studied in a reproducible laser-axotomy model successfully established on primary hippocampal neurons isolated from newborn BALB/c mice. Delivered within CurcuEmulsomes, curcumin promoted cell survival after the laser injury. Immunocytochemistry analyses revealed significantly higher 
expression levels for Bcl2 when injured neurons were treated with CurcuEmulsome or curcumin compared to the untreated injured control group $(P \leq 0.05)$. Caspase 3 expression levels decreased when treated with CurcuEmulsome or curcumin. However, the neuroprotective effect of CurcuEmulsomes remained limited, which is attributed to the sustained release profile of the emulsomes yielding lower concentrations of curcumin actively present within the cytoplasm. Minor changes in expression levels of Wnt3a and mTOR were observed for both CurcuEmulsome- and curcumin-treated injured neurons compared to the values for the untreated injured control group.

Concisely, with their safety profile and the biological effects on the studied in vitro axotomy model of primary hippocampal neurons, CurcuEmulsomes demonstrate potential to achieve neuroprotection against neuronal injuries in the CNS. Our future research will include in vivo studies where the neuroprotective effect of the formulation and its permeability through the BBB will be the focus, and the biggest challenge in clinical applications.

\section{Abbreviations}

$\mathrm{AD}$, Alzheimer's disease; AKT, protein kinase B (PKB); ALS, amyotrophic lateral sclerosis; BBB, blood-brain barrier; Bcl2, B-cell lymphoma 2; BSA, bovine serum albumin; CGN, cerebellar granule neuron; CLSM, confocal laser scanning microscope; CNS, central nervous system; DAPI, 4',6-diamidino-2-phenylindole; DiI, 1,1'-dioctadecyl-3,3,3',3'-tetramethylindocarbocyanine perchlorate; DIV, day in vitro; DLS, dynamic light scattering; DMSO, dimethyl sulfoxide; DNAse I, deoxyribonuclease I; DPPC, 1.2-dipalmitoyl-rac-glycero -3-phosphocholine; DRG, dorsal root ganglion; FBS, fetal bovine serum; GSK3b, glycogen synthase kinase 3 beta; HCT116, a human colon cancer cell line; HepG2, a human liver cancer cell line; IAA, iodoacetamide; ICC, immunocytochemistry; IgG, immunoglobulin G; LLC-PK1, epitheliallike pig kidney cell line; LNCaP, cell line derived from a metastatic lymph-node lesion of human prostate cancer; MCF7, a human breast adenocarcinoma cell line; mTOR, mammalian target of rapamycin; mTORC, mammalian target of rapamycin complex; MTS, 3-(4,5-dimethylthiazol-2-yl)5-(3-carboxymethoxyphenyl)-2-(4-sulfophenyl)-2h-tetrazolium; NADH, reduced nicotinamide adenine dinucleotide; $\mathrm{NADPH}$, reduced nicotinamide adenine dinucleotide phosphate; NBA, neurobasal-A; ND, neurodegenerative disease; NMDA, N-methyl-D-aspartate; P0, postnatal day zero; PBS, phosphate-buffered saline; PD, Parkinson's disease; PDI, polydispersity index; PES, phenazine ethosulfate; PI, propidium iodide; PI3K, phosphoinositide 3-kinase; ROS, reactive oxygen species; SEM, standard error of the mean; TBS, Tris-buffered saline; WHO, World Health Organization; Wnt3a, wingless-type MMTV integration site family, member $3 \mathrm{~A}$.

\section{Ethics Approval and Informed Consent}

Animal experiments were conducted in accordance with the European Community guidelines and approved by Istanbul Medipol University Ethics Committee for Animal Experimentation (date: 07/03/2016; approval number: 38828770-604.01.01-E.3722).

\section{Acknowledgments}

Financial support from the Scientific and Technological Research Council of Turkey (TÜBITTAK) under project number $116 \mathrm{Z} 347$ is gratefully acknowledged. Dynamic light scattering analysis was carried out using a Zetasizer instrument (Nano ZS, Malvern Instruments Ltd, UK) in the Genetics and Bioengineering Department at Yeditepe University. The authors would like to thank to Dr Bilal Kerman from Istanbul Medipol University and Dr Aysegul Yildiz from Mugla Sitki Kocman University for their important remarks and feedback on the experimental framework. The authors would also like to express their gratitude to Dr Florence Johnson for her kind assistance in the proof-reading of the manuscript.

\section{Author Contributions}

EY produced CurcuEmulsomes and performed the related characterization experiments under supervision of MU. $\mathrm{EY}$ and SB isolated hippocampal neurons from newborn $\mathrm{BALB} / \mathrm{c}$ mice. EY performed primary cell culture studies including cellular uptake and cell viability. EY and SB executed laser axotomy, confocal microscopy analysis and immunocytochemistry analysis. EY produced the quantitative data from the fluorescence images. Together with GO, MU designed the framework of the study, provided the funding, guided the conduct of studies, and supervised data analysis. All authors have interpreted the data; drafted, written, and critically reviewed the article. The original and revised drafts were written through contributions of all authors. All authors have given approval to the final version of the manuscript to be published, agreed on the journal to which the article 
will be submitted and agreed to be accountable for all aspects of the work.

\section{Funding}

Financial support from the Scientific and Technological Research Council of Turkey (TÜBİTAK) under project number $116 Z 347$ is gratefully acknowledged.

\section{Disclosure}

The authors report no conflicts of interest in this work.

\section{References}

1. Gammon K. Neurodegenerative disease: brain windfall. Nature. 2014;515(7526):299-300. doi:10.1038/nj7526-299a

2. Salvadores N, Sanhueza M, Manque P, Court FA. Axonal degeneration during aging and its functional role in neurodegenerative disorders. Front Neurosci. 2017;11. doi:10.3389/fnins.2017.00451

3. Kennedy BK, Berger SL, Brunet A, et al. Geroscience: linking aging to chronic disease. Cell. 2014;159(4):709-713. doi:10.1016/j. cell.2014.10.039

4. Sharifi-Rad M, Lankatillake C, Dias DA, et al. Impact of Natural Compounds on Neurodegenerative Disorders: from Preclinical to Pharmacotherapeutics. J Clin Med. 2020. doi:10.3390/jcm9041061

5. Neukomm LJ, Freeman MR. Diverse cellular and molecular modes of axon degeneration. Trends Cell Biol. 2014;24(9):515-523. doi:10.1016/j.tcb.2014.04.003

6. Feinberg K, Kolaj A, Wu C, et al. A neuroprotective agent that inactivates prodegenerative TrkA and preserves mitochondria J Cell Biol. 2017;216(11):3655-3675. doi:10.1083/jcb.201705085

7. Wang Y, Song M, Song F. Neuronal autophagy and axon degeneration. Cell Mol Life Sci. 2018;75(13):2389-2406. doi:10.1007/s00018-018-2812-1

8. Burke RE, O'Malley K. Axon degeneration in Parkinson's disease. Exp Neurol. 2013;246:72-83. doi:10.1016/j.expneurol.2012.01.011

9. Uliassi E, Gandini A, Perone RC, Bolognesi ML. Neuroregeneration versus neurodegeneration: toward a paradigm shift in Alzheimer's disease drug discovery. Future Med Chem. 2017;9(10):995-1013. doi:10.4155/fmc-2017-0038

10. Goldfrank L, Lewin N, Flomenbaum N, Howland MA. The pernicious panacea: herbal medicine. Hosp Physician. 1982.

11. Cowan MM. Plant products as antimicrobial agents. Clin Microbiol Rev. 1999;12(4):564-582. doi:10.1128/cmr.12.4.564

12. Thiyagarajan M, Sharma SS. Neuroprotective effect of curcumin in middle cerebral artery occlusion induced focal cerebral ischemia in rats. Life Sci. 2004;74(8):969-985. doi:10.1016/j.1fs.2003.06.042

13. Lim GP, Chu T, Yang F, Beech W, Frautschy SA, Cole GM. The curry spice curcumin reduces oxidative damage and amyloid pathology in an Alzheimer transgenic mouse. J Neurosci. 2001;21 (21):8370-8377. doi:10.1523/jneurosci.21-21-08370.2001

14. Mohammadi S, Jafari B, Asgharian P, Martorell M, Sharifi-Rad J. Medicinal plants used in the treatment of Malaria: A key emphasis to Artemisia, Cinchona, Cryptolepis, and Tabebuia genera. Phyther Res. 2020. doi:10.1002/ptr.6628

15. Islam MT, Hossain R, Hassan SMH, et al. Biological activities of sinularin: A literature-based review. Cell Mol Biol. 2020. doi:10.14715/cmb/2020.66.4.6

16. Bagheri G, Ayatollahi SA, Ramírez-Alarcón K, et al. Phytochemical screening of Alstonia scholaris leaf and bark extracts and their antimicrobial activities. Cell Mol Biol. 2020. doi:10.14715/cmb/ 2020.66.4.32
17. Salehi B, Zakaria ZA, Gyawali R, et al. Piper species: A comprehensive review on their phytochemistry, biological activities and applications. Molecules. 2019;24:7. doi:10.3390/molecules24071364

18. Yang F, Lim GP, Begum AN, et al. Curcumin inhibits formation of amyloid $\beta$ oligomers and fibrils, binds plaques, and reduces amyloid in vivo. $J$ Biol Chem. 2005;280(7):5892-5901. doi:10.1074/jbc. M404751200

19. Huang H-C, Zheng B-W, Guo Y, et al. Antioxidative and Neuroprotective Effects of Curcumin in an Alzheimer's Disease Rat Model Co-Treated with Intracerebroventricular Streptozotocin and Subcutaneous D-Galactose. J Alzheimer's Dis. 2016;52(3):899-911. doi:10.3233/JAD-150872

20. Motaghinejad M, Motevalian M, Fatima S, Hashemi H, Gholami M. Curcumin confers neuroprotection against alcohol-induced hippocampal neurodegeneration via CREB-BDNF pathway in rats. Biomed Pharmacother. 2017;87:721-740. doi:10.1016/j.biopha.2016.12.020

21. Wu J, Li Q, Wang X, et al. Neuroprotection by Curcumin in Ischemic Brain Injury Involves the Akt/Nrf2 Pathway. PLoS One. 2013;8:3. doi:10.1371/journal.pone. 0059843

22. Joe B, Vijaykumar M, Lokesh BR. Biological Properties of Curcumin-Cellular and Molecular Mechanisms of Action. Crit Rev Food Sci Nutr. 2004;44(2):97-111. doi:10.1080/10408690490424702

23. Mythri B, Srinivas Bharath RM, Curcumin M. A Potential Neuroprotective Agent in Parkinson's Disease. Curr Pharm Des. 2012;18(1):91-99. doi:10.2174/138161212798918995

24. Salehi B, Ata A, Kumar NVA, et al. Antidiabetic potential of medicinal plants and their active components. Biomolecules. 2019. doi:10.3390/biom9100551

25. Islam MT, Hasan J, Snigdha HMSH, et al. Chemical profile, traditional uses, and biological activities of Piper chaba Vahl: A review. J Ethnopharmacol. 2020:257. doi:10.1016/j.jep.2020.112853.

26. Dong S, Zeng Q, Mitchell ES, et al. Curcumin enhances neurogenesis and cognition in aged rats: implications for transcriptional interactions related to growth and synaptic plasticity. PLoS One. 2012;7:2. doi:10.1371/journal.pone.0031211

27. Zhao J, Yu S, Zheng W, et al. Curcumin improves outcomes and attenuates focal cerebral ischemic injury via antiapoptotic mechanisms in rats. Neurochem Res. 2010;35(3):374-379. doi:10.1007/ s11064-009-0065-y

28. Salehi B, Calina D, Docea A, et al. Curcumin's Nanomedicine Formulations for Therapeutic Application in Neurological Diseases. J Clin Med. 2020. doi:10.3390/jcm9020430

29. Frautschy SA, Hu W, Kim P, et al. Phenolic anti-inflammatory antioxidant reversal of $\mathrm{A} \beta$-induced cognitive deficits and neuropathology. Neurobiol Aging. 2001;22(6):993-1005. doi:10.1016/S0197-4580(01)00300-1

30. Ishrat T, Hoda MN, Khan MB, et al. Amelioration of cognitive deficits and neurodegeneration by curcumin in rat model of sporadic dementia of Alzheimer's type (SDAT). Eur Neuropsychopharmacol. 2009;19(9):636-647. doi:10.1016/j.euroneuro.2009.02.002

31. Lichtenwalner RJ, Parent JM. Adult neurogenesis and the ischemic forebrain. J Cereb Blood Flow Metab. 2006;26(1):1-20. doi:10.1038/ sj.jcbfm. 9600170

32. Ma QL, Zuo X, Yang F, et al. Curcumin suppresses soluble Tau dinners and corrects molecular chaperone, synaptic, and behavioral deficits in aged human Tau transgenic mice. J Biol Chem. 2013;288 (6):4056-4065. doi:10.1074/jbc.M112.393751

33. Tiwari SK, Agarwal S, Seth B, et al. Curcumin-loaded nanoparticles potently induce adult neurogenesis and reverse cognitive deficits in Alzheimer's disease model via canonical Wnt/ $\beta$-catenin pathway. ACS Nano. 2014;8(1):76-103. doi:10.1021/nn405077y

34. Chung S, Yao H, Caito S. Regulation of SIRT1 in cellular functions: role of polyphenols. Arch Biochem Biophys. 2010;501(1):79-90. doi:10.1016/j.abb.2010.05.003

35. Reuter S, Gupta SC, Park B, Goel A, Aggarwal BB. Epigenetic changes induced by curcumin and other natural compounds. Genes Nutr. 2011;6(2):93-108. doi:10.1007/s12263-011-0222-1 
36. Zhao C, Deng W, Gage FH. Mechanisms and Functional Implications of Adult Neurogenesis. Cell. 2008;132(4):645-660. doi:10.1016/j. cell.2008.01.033

37. Cole GM, Teter B, Frautschy SA. Neuroprotective effects of curcumin. Adv Exp Med Biol. 2007;595:197-212. doi:10.1007/9780-387-46401-5_8

38. Ringman J, Frautschy S, Cole G, Masterman D, Cummings J, Potential A. Role of the Curry Spice Curcumin in Alzheimers Disease. Curr Alzheimer Res. 2005;2(2):131-136. doi:10.2174/ 1567205053585882

39. Kaminaga Y, Nagatsu A, Akiyama T, et al. Production of unnatural glucosides of curcumin with drastically enhanced water solubility by cell suspension cultures of Catharanthus roseus. FEBS Lett. 2003;555 (2):311-316. doi:10.1016/S0014-5793(03)01265-1

40. Schiborr C, Eckert GP, Rimbach G, Frank J. A validated method for the quantification of curcumin in plasma and brain tissue by fast narrow-bore high-performance liquid chromatography with fluorescence detection. Anal Bioanal Chem. 2010;397(5):1917-1925. doi:10.1007/s00216-010-3719-3

41. Sharma RA, Euden SA, Platton SL, et al. Phase I clinical trial of oral curcumin: biomarkers of systemic activity and compliance. Clin Cancer Res. 2004;10(20):6847-6854. doi:10.1158/1078-0432.CCR04-0744

42. Shoba G, Joy D, Joseph T, Majeed M, Rajendran R, Srinivas P. Influence of piperine on the pharmacokinetics of curcumin in animals and human volunteers. Planta Med. 1998;64(04):353-356.

43. Asai A, Miyazawa T. Occurrence of orally administered curcuminoid as glucuronide and glucuronide/sulfate conjugates in rat plasma. Life Sci. 2000;67(23):2785-2793. doi:10.1016/S0024-3205(00)00868-7

44. Anand P, Kunnumakkara AB, Newman RA, Aggarwal BB. Bioavailability of curcumin: problems and promises. Mol Pharm. 2007;4(6):807-818. doi:10.1021/mp700113r

45. Pan MH, Huang TM, Lin JK. Biotransformation of curcumin through reduction and glucuronidation in mice. Drug Metab Dispos. 1999;27 (4):486-494

46. Purkayastha S, Berliner A, Fernando SS, et al. Curcumin blocks brain tumor formation. Brain Res. 2009;1266:130-138. doi:10.1016/j. brainres.2009.01.066

47. Yallapu MM, Nagesh PKB, Jaggi M, Chauhan SC. Therapeutic Applications of Curcumin Nanoformulations. AAPS J. 2015;17 (6):1341-1356. doi:10.1208/s12248-015-9811-z

48. Tsai YM, Chien CF, Lin LC, Tsai TH. Curcumin and its nano-formulation: the kinetics of tissue distribution and blood-brain barrier penetration. Int J Pharm. 2011;416(1):331-338. doi:10.1016/j. ijpharm.2011.06.030

49. Barbara R, Belletti D, Pederzoli F, et al. Novel Curcumin loaded nanoparticles engineered for Blood-Brain Barrier crossing and able to disrupt Abeta aggregates. Int J Pharm. 2017;526(1-2):413-424. doi:10.1016/j.ijpharm.2017.05.015

50. Cheng KK, Yeung CF, Ho SW, Chow SF, Chow AHL, Baum L. Highly stabilized curcumin nanoparticles tested in an in vitro bloodbrain barrier model and in alzheimer's disease Tg2576 Mice. AAPS J. 2013;15(2):324-336. doi:10.1208/s12248-012-9444-4

51. Ucisik MH, Küpcü S, Schuster B, Sleytr UB. Characterization of CurcuEmulsomes: nanoformulation for enhanced solubility and delivery of curcumin. J Nanobiotechnol. 2013;11:1. doi:10.1186/ 1477-3155-11-37

52. Bolat ZB, Islek Z, Demir BN, Yilmaz EN, Sahin F, Ucisik MH. Curcumin- and Piperine-Loaded Emulsomes as Combinational Treatment Approach Enhance the Anticancer Activity of Curcumin on HCT116 Colorectal Cancer Model. Front Bioeng Biotechnol. 2020;8. doi:10.3389/fbioe.2020.00050

53. Bisht S, Feldmann G, Soni S, et al. Polymeric nanoparticleencapsulated curcumin ("nanocurcumin"): A novel strategy for human cancer therapy. J Nanobiotechnology. 2007:5. doi:10.1186/ 1477-3155-5-3.
54. Anitha A, Deepagan VG, Divya Rani VV, Menon D, Nair SV, Jayakumar R. Preparation, characterization, in vitro drug release and biological studies of curcumin loaded dextran sulphate-chitosan nanoparticles. Carbohydr Polym. 2011;84(3):1158-1164. doi:10. 1016/j.carbpol.2011.01.005

55. Levenfus I. An Efficient Method for Counting DAPI-Stained Cells Using Fiji. GRIN Verlag. 2011. http://content.grin.com/document/ v168646.pdf..

56. Ucisik MH, Küpcü S, Debreczeny M, Schuster B, Sleytr UB. S-layer coated emulsomes as potential nanocarriers. Small. 2013;9 (17):2895-2904. doi:10.1002/smll.201203116

57. Bernardi A, Frozza RL, Meneghetti A, et al. Indomethacin-loaded lipid-core nanocapsules reduce the damage triggered by $A \beta 1-42$ in Alzheimer's disease models. Int J Nanomedicine. 2012;7:4927-4942. doi:10.2147/IJN.S35333

58. Ianiski FR, Alves CB, Souza ACG, et al. Protective effect of meloxicam-loaded nanocapsules against amyloid- $\beta$ peptide-induced damage in mice. Behav Brain Res. 2012;230(1):100-107. doi:10. 1016/j.bbr.2012.01.055

59. Frozza RL, Bernardi A, Hoppe JB, et al. Neuroprotective effects of resveratrol against $A \beta$ administration in rats are improved by lipidcore nanocapsules. Mol Neurobiol. 2013;47(3):1066-1080. doi:10.1007/s12035-013-8401-2

60. Hoppe JB, Coradini K, Frozza RL, et al. Free and nanoencapsulated curcumin suppress $\beta$-amyloid-induced cognitive impairments in rats: involvement of BDNF and Akt/GSK-3 $\beta$ signaling pathway. Neurobiol Learn Mem. 2013;106:134-144. doi:10.1016/j.nlm.2013. 08.001

61. Dimer FA, Pigatto MC, Boque CA, et al. Nanoencapsulation improves relative bioavailability and antipsychotic effect of olanzapine in rats. J Biomed Nanotechnol. 2015;11(8):1482-1493. doi:10. 1166/jbn.2015.2082

62. Giacomeli R, Izoton JC, Santos RB. Neuroprotective effects of curcumin lipid-core nanocapsules in a model Alzheimer's disease induced by $\beta$-amyloid 1-42 peptide in aged female mice. Brain Res. 2019. doi:10.1016/j.brainres.2019.146325

63. Garza-Lombó C, Gonsebatt ME. Mammalian target of rapamycin: its role in early neural development and in adult and aged brain function. Front Cell Neurosci. 2016;10(JUN). doi:10.3389/fncel.2016.00157

64. Peng Y, Jiang B, Wu H, Dai R, Tan L. Effects of genistein on neuronal apoptosis, and expression of Bcl-2 and Bax proteins in the hippocampus of ovariectomized rats. Neural Regen Res. 2012;7 (36):2874-2881. doi:10.3969/j.issn.1673-5374.2012.36.004

65. Kamada S, Kikkawa U, Tsujimoto Y, Hunter T. Nuclear translocation of caspase-3 is dependent on its proteolytic activation and recognition of a substrate-like protein(s). J Biol Chem. 2005;280(2):857-860. doi:10.1074/jbc.C400538200

66. Ucisik M, Sleytr U, Schuster B. Emulsomes Meet S-layer Proteins: an Emerging Targeted Drug Delivery System. Curr Pharm Biotechnol. 2015;16(4):392-405. doi:10.2174/138920101604150 218112656

67. Tønnesen HH, Karlsen J. Studies on curcumin and curcuminoids V. Alkaline Degradation of Curcumin. Z Lebensm Unters Forsch. 1985;180(2):132-134. doi:10.1007/BF01042637

68. Sahay G, Alakhova DY, Kabanov AV. Endocytosis of nanomedicines. $J$ Control Release. 2010;145(3):182-195. doi:10.1016/j.jconrel.2010. 01.036

69. Singh R, Tønnesen HH, Kristensen S, Berg K. The influence of Pluronics ${ }^{\circledR}$ on dark cytotoxicity, photocytotoxicity, localization and uptake of curcumin in cancer cells: studies of curcumin and curcuminoids XLIX. Photochem Photobiol Sci. 2013;12(3):559-575. doi:10.1039/c2pp25249j

70. So JK, Tae GS, Hee RP, et al. Curcumin stimulates proliferation of embryonic neural progenitor cells and neurogenesis in the adult hippocampus. J Biol Chem. 2008;283(21):14497-14505. doi:10.10 74/jbc.M708373200 
71. Ray B, Bisht S, Maitra A, Maitra A, Lahiri DK. Neuroprotective and neurorescue effects of a novel polymeric nanoparticle formulation of curcumin (NanoCurcTM) in the neuronal cell culture and animal model: implications for Alzheimer's disease. J Alzheimer's Dis. 2011;23(1):61-77. doi:10.3233/JAD-2010-101374

72. Cohly HHP, Taylor A, Angel MF, Salahudeen AK. Effect of turmeric, turmerin and curcumin on $\mathrm{H} 2 \mathrm{O} 2$-induced renal epithelial (LLC-PK1) cell injury. Free Radic Biol Med. 1998;24(1):49-54. doi:10.1016/ S0891-5849(97)00140-8

73. Reyes-Fermín LM, González-Reyes S, Tarco-Álvarez NG, Hernández-Nava M, Orozco-Ibarra M, Pedraza-Chaverri J Neuroprotective effect of $\alpha$-mangostin and curcumin against iodoacetate-induced cell death. Nutr Neurosci. 2012;15(5):34-41. doi:10.1179/1476830512Y.0000000011

74. Srivastava P, Dhuriya YK, Kumar V, et al. PI3K/Akt/GSK3 $\beta$ induced CREB activation ameliorates arsenic mediated alterations in NMDA receptors and associated signaling in rat hippocampus: neuroprotective role of curcumin. Neurotoxicology. 2018. doi:10.1016/j. neuro.2018.04.018

75. Cengiz N, Öztürk G, Erdoğan E, Him A, Oğuz EK. Consequences of neurite transection in vitro. J Neurotrauma. 2012;29(15):2465-2474. doi:10.1089/neu.2009.0947

76. Pérez-Palma E, Andrade V, Caracci MO, et al. Early transcriptional changes induced by $\mathrm{Wnt} / \beta$-catenin signaling in hippocampal neurons. Neural Plast. 2016:2016. doi:10.1155/2016/4672841.

77. Pinnock SB, Blake AM, Platt NJ, Herbert J. The roles of BDNF, pCREB and wnt3a in the latent period preceding activation of progenitor cell mitosis in the adult dentate Gyrus by fluoxetine. PLoS One. 2010;5:10. doi:10.1371/journal.pone.0013652

78. Spaccapelo L, Galantucci M, Neri L, et al. Up-regulation of the canonical Wnt-3A and Sonic hedgehog signaling underlies melanocortin-induced neurogenesis after cerebral ischemia. Eur J Pharmacol. 2013;707(13):78-86. doi:10.1016/j.ejphar.2013.03.030

79. Stankowska DL, Krishnamoorthy VR, Ellis DZ, Krishnamoorthy RR. Neuroprotective effects of curcumin on endothelin-1 mediated cell death in hippocampal neurons. Nutr Neurosci. 2017;20(5):273-283. doi:10.1080/1028415X.2015.1119377

80. Patel AK, Park KK, Hackam AS. Wnt signaling promotes axonal regeneration following optic nerve injury in the mouse. Neuroscience. 2017;343:372-383. doi:10.1016/j.neuroscience.2016. 12.020

81. Seitz R, Hackl S, Seibuchner T, Tamm ER, Ohlmann A. Norrin mediates neuroprotective effects on retinal ganglion cells via activation of the Wnt/ $\beta$-catenin signaling pathway and the induction of neuroprotective growth factors in Müller cells. J Neurosci. 2010;30 (17):5998-6010. doi:10.1523/JNEUROSCI.0730-10.2010
82. Liu Y, Wang X, Lu CC, et al. Repulsive Wnt signaling inhibits axon regeneration after CNS injury. J Neurosci. 2008;28(33):8376-8382. doi:10.1523/JNEUROSCI.1939-08.2008

83. Hollis ER, Zou Y. Reinduced Wnt signaling limits regenerative potential of sensory axons in the spinal cord following conditioning lesion. Proc Natl Acad Sci U S A. 2012;109(36):14663-14668. doi:10.1073/pnas.1206218109

84. Yin ZS, Zu B, Chang J, Zhang H. Repair effect of Wnt3a protein on the contused adult rat spinal cord. Neurol Res. 2008;30(5):480-486. doi:10.1179/174313208X284133

85. David MD, Cantí C, Herreros J. Wnt-3a and Wnt-3 differently stimulate proliferation and neurogenesis of spinal neural precursors and promote neurite outgrowth by canonical signaling. J Neurosci Res. 2010;88(14):3011-3023. doi:10.1002/jnr.22464

86. Guo JN, Tian LY, Liu WY, Mu J, Zhou D. Activation of the Akt/ mTOR signaling pathway: A potential response to long-term neuronal loss in the hippocampus after sepsis. Neural Regen Res. 2017;12 (11):1832-1842. doi:10.4103/1673-5374.219044

87. Beevers C, Zhou H, Huang S. Hitting the Golden TORget: curcumin's Effects on mTOR Signaling. Anticancer Agents Med Chem. 2013;13(7):988-994. doi:10.2174/1871520611313070004

88. Marie Hardwick J, Soane L. Multiple functions of BCL-2 family proteins. Cold Spring Harb Perspect Biol. 2013;5:2. doi:10.1101/ cshperspect.a008722

89. Green DR, Reed JC. Mitochondria and apoptosis. Science. 1998;281 (5381):1309-1312.

90. Howard S, Bottino C, Brooke S, Cheng E, Giffard RG, Sapolsky R. Neuroprotective effects of bcl-2 overexpression in hippocampal cultures: interactions with pathways of oxidative damage. J Neurochem. 2002;83(4):914-923. doi:10.1046/j.1471-4159.2002.01198.x

91. Tiwari SK, Agarwal S, Tripathi A, Chaturvedi RK. Bisphenol-A Mediated Inhibition of Hippocampal Neurogenesis Attenuated by Curcumin via Canonical Wnt Pathway. Mol Neurobiol. 2016;53 (5):3010-3029. doi:10.1007/s12035-015-9197-z

92. Zhao M, Su J, Head E, Cotman CW. Accumulation of caspase cleaved amyloid precursor protein represents an early neurodegenerative event in aging and in Alzheimer's disease. Neurobiol Dis. 2003;14(3):391-403. doi:10.1016/j.nbd.2003.07.006

93. Mattson MP. Apoptosis in neurodegenerative disorders. Nat Rev Mol Cell Biol. 2000;1(2):120-129. doi:10.1097/yco.0000000000000476

94. Wang Q, Sun AY, Simonyi A, et al. Neuroprotective mechanisms of curcumin against cerebral ischemia-induced neuronal apoptosis and behavioral deficits. $J$ Neurosci Res. 2005;82(1):138-148. doi: 10.1002/jnr.20610
International Journal of Nanomedicine

\section{Publish your work in this journal}

The International Journal of Nanomedicine is an international, peerreviewed journal focusing on the application of nanotechnology in diagnostics, therapeutics, and drug delivery systems throughout the biomedical field. This journal is indexed on PubMed Central, MedLine, CAS, SciSearch ${ }^{\circledR}$, Current Contents ${ }^{\circledR} /$ Clinical Medicine,
Journal Citation Reports/Science Edition, EMBase, Scopus and the Elsevier Bibliographic databases. The manuscript management system is completely online and includes a very quick and fair peer-review system, which is all easy to use. Visit http://www.dovepress.com/ testimonials.php to read real quotes from published authors. 